\title{
Chromatin Higher-order Structure and Dynamics
}

\author{
Christopher L. Woodcock ${ }^{1,2}$ and Rajarshi P. Ghosh ${ }^{2}$ \\ ${ }^{1}$ Biology Department, University of Massachusetts, Amherst, Massachusetts 01003 \\ ${ }^{2}$ Program in Molecular and Cellular Biology, University of Massachusetts, Amherst, Massachusetts 01003 \\ Correspondence: chris@bio.umass.edu
}

The primary role of the nucleus as an information storage, retrieval, and replication site requires the physical organization and compaction of meters of DNA. Although it has been clear for many years that nucleosomes constitute the first level of chromatin compaction, this contributes a relatively small fraction of the condensation needed to fit the typical genome into an interphase nucleus or set of metaphase chromosomes, indicating that there are additional "higher order" levels of chromatin condensation. Identifying these levels, their interrelationships, and the principles that govern their occurrence has been a challenging and much discussed problem. In this article, we focus on recent experimental advances and the emerging evidence indicating that structural plasticity and chromatin dynamics play dominant roles in genome organization. We also discuss novel approaches likely to yield important insights in the near future, and suggest research areas that merit further study.

\begin{abstract}
$A s$ the cell's primary information storage, Aretrieval, and duplication organelle, the nucleus, by analogy with human-created information repositories (libraries and computer discs), might be expected to be highly structured. However, chromatin within the living nucleus appears to be organized in a quite unstructured manner, and the rules that define or encode the principles of chromatin organization have been difficult to decipher. These frustrations are reflected in some reviews of chromatin organization with titles such as "Chromatin higher-order structure: chasing a mirage?" (van Holde and Zlatanova 1995), "Higher-order structures of chromatin: the elusive $30 \mathrm{~nm}$ fiber" (Tremethick 2007), and "Chromatin fiber structure: where is the problem now?" (van Holde and Zlatanova 2007). In this article, we discuss what is known about chromatin structure, and
\end{abstract}

consider the prospects of improving our understanding in the near future. In recent years, work on chromatin organization has increasingly focused on the many dynamic aspects of chromatin, which contribute to its structural and functional plasticity, and it is becoming clear that dynamics plays a crucially important functional role, perhaps contributing to nuclear selforganization (Misteli 2001).

\section{CHROMATIN HIGHER-ORDER STRUCTURE}

In the context of chromatin, "higher-order structure" may be defined as any assemblage of nucleosomes that assumes a reproducible conformation in 3D space. The most obvious chromatin higher-order structure is the mitotic/ meiotic chromosome in which the DNA is compacted some 10,000- to 20,000-fold. Metaphase

Editors: David Spector and Tom Misteli

Additional Perspectives on The Nucleus available at www.cshperspectives.org

Copyright (C) 2010 Cold Spring Harbor Laboratory Press; all rights reserved; doi: 10.1101/cshperspect.a000596

Cite this article as Cold Spring Harb Perspect Biol 2010;2:a000596 
chromosomes have characteristic shapes, banding patterns, and locations of specific genes. Although chromosomes have a consistent structure at the light microscope level in terms of longitudinal positioning of bands and genes, this consistency is evidently modulated by an intrinsic variability in longitudinal position of up to $0.3 \mu \mathrm{m}$ (Strukov and Belmont 2009). However, these authors reported that there was no consistency in the axial positioning of loci, suggesting that chromosome architecture involves considerable plasticity at some level or levels of folding. Understanding how chains of nucleosomes are folded in the creation of mitotic chromosomes and how they are arranged in interphase continues to be an exciting yet technically challenging endeavor.

The concept of primary, secondary, tertiary, and quaternary structures used for proteins can also be usefully applied to chromatin structural hierarchies (Woodcock and Dimitrov 2001), with the beads-on-a-string organization of nucleosomes and linker DNA constituting the primary structure, and arrangements resulting from interactions between nucleosomes giving rise to secondary structures. Thus, the chromatin equivalent of protein secondary structure might involve interactions analogous to those leading to $\alpha$-helices and $\beta$-sheets. Unlike proteins that consist of sequences of 20 amino acids, chromatin consists of a repeating chain of more-or-less identical nucleosomes, and thus might be predicted to form highly "ordered" secondary structures. Note that the term "ordered" can be used in the sense that a crystal is highly ordered and also used to describe hierarchical levels where the concept is more akin to its meaning in "orders of magnitude."

Although in one sense, the basic nucleosome-linker DNA unit comprising the primary structure of chromatin is simple, there are a number of potentially variable parameters that contribute to complexity. There is structural uncertainty even at the level of the nucleosome core particle (NCP). Although the structure of the NCP is well established at the atomic level (Luger et al. 1997), portions of the four core histones are not seen in crystals because of their evident mobility. For example, the unstructured terminal regions that are extremely important in modulating chromatin structure are not seen in X-ray data. The length of linker DNA between NCPs varies not only between species, but also between tissues of the same organism, and within a single nucleus (van Holde 1989). Mean linker lengths (defined as the nucleosome repeat length minus the $\sim 145$ bp of DNA protected in NCPs) range from a low value of $\sim 20$ bp in budding yeast to $\sim 75$ bp in echinoderm sperm. For a typical vertebrate nucleus the mean linker length is $\sim 35 \mathrm{bp}$. The rich literature concerning the locations of nucleosome positions on specific DNA sequences, reveals instances, especially in the upstream control regions of genes, in which nucleosomes are preferentially located or excluded, and this positioning is often important in regulating transcription (Simpson 1991; Jiang and Pugh 2009). For some DNA sequences, the preference for nucleosomes to be present at a particular location is retained when the nucleosomes are reconstituted on the DNA in vitro. In addition to linker DNA length, nucleosomes vary in their complement of core histone variants and histone postsynthetic modifications. All these variations are likely to impact chromatin secondary structures.

The obvious experimental approaches to determine chromatin secondary and higherorder structures using light and electron microscopy yield disappointingly little information, largely because nucleosomes and linker DNA cannot be adequately resolved in the compact chromatin that occurs in the nucleus. A recent study using cryo-electron microscopy of thin sections of chromosomes vitrified in vivo, and therefore expected to faithfully reflect the native structure, provides an instructive example. Despite careful image processing of the micrographs, there was no evidence for any higher-order structure, leading the authors to suggest that the chromatin forms a "molten" mass similar to that assumed by certain polymers, and that in this form, the size, shape and trajectories of any higher-order structures would not be resolved (Eltsov et al. 2008). This may well be the case, but nevertheless, the arrangements of arrays of nucleosomes in 
3D space are to some extent constrained and nonrandom, and important information would be obtained if it were possible to trace the paths of chains of nucleosomes through the nucleus. The generally amorphous appearance of chromatin in thin sections contrasts sharply with the appearance of cytoskeletal elements such as microtubules, which can be resolved in cells even when in compact bundles. This striking difference supports the concept that tightly packed chains of nucleosomes readily interdigitate with each other, leading to the rather featureless appearance of chromatin in EM images (Woodcock and Horowitz 1995; Woodcock 2006). A new technique for preparing frozen hydrated material that involves thinning of samples by milling with a focused ion beam (Marko et al. 2007, 2008) that does not involve the compression and possible local heating of cryosectioning is currently under development in several laboratories, and it will be most interesting to see whether this method provides improved structural information when applied to nuclear structures.

\section{The 30-nm Fiber}

In the absence of clear conclusions from the examination of nuclei and chromosomes in situ, many investigators have focused on isolated chromatin. Through the use of nucleases that cut the linker DNA between nucleosomes, it is possible to isolate polynucleosomes and study their properties under defined conditions in solution. Early work clearly established that the compaction state of polynucleosomes is highly dependent on the ionic milieu. Naked DNA, being highly charged, is self-repulsive in low ionic strength buffers-the self-repulsion can be reduced or eliminated in the presence of cations, with divalent and polyvalent cations being especially effective. Chromatin behaves similarly to DNA in this respect because the DNA negative charge is not fully neutralized by histones. The response of chains of nucleosomes to changes in the ionic environment can be satisfactorily modeled (Clark and Kimura 1990; Arya and Schlick 2006), suggesting that our basic understanding of the response of
Chromatin Higher-order Structure and Dynamics

chromatin to changes in the ionic environment is robust.

Electron microscopy of isolated polynucleosomes clearly reveals an open beads-on-a-string conformation in low salt and a progressive compaction that occurs as the ionic strength is raised. There is general agreement that an early stage in compaction is the formation of a fiber $\sim 30 \mathrm{~nm}$ in diameter, and that, at least for polynucleosomes in vitro, this constitutes a bona fide chromatin secondary structure. However, the arrangement of nucleosomes and linker DNA within isolated $30 \mathrm{~nm}$ fibers has been difficult to study and remains controversial. Careful examination of isolated $30 \mathrm{~nm}$ fibers has failed to reveal any consistent clear-cut arrangement of nucleosomes. The realization that native nucleosomes are not identical, but, as described earlier, vary in several parameters, suggested that these could be the source of the equivocal findings regarding $30-\mathrm{nm}$ fiber organization. To eliminate these potential problems, investigators turned to fully defined artificial polynucleosomes reconstituted onto DNA containing regularly spaced nucleosome localization sequences (Simpson et al. 1985). A major achievement using this approach was the successful crystallization and structure determination by X-ray diffraction of a tetranucleosome (Schlach et al. 2005). This showed unequivocally that, at least for this particular construct, exposed to crystallization-promoting conditions that included $90-\mathrm{mM} \mathrm{Mg}^{++}$, the complex assumed a zigzag arrangement, with contacts between nucleosomes 1 and 3 and between 2 and 4, with the linker extending between them (Fig. 1). The work clearly established the predominant internucleosome interactions that result in a specific secondary structure for this particular chromatin construct in this ionic environment. It is not yet clear how far the zigzag structure can be extrapolated to long chromatin with heterogeneity in histone content and linker length. Nevertheless, it does provide an important context in which to view earlier results. For example, the interpretation of the first EM studies of isolated compact $30-\mathrm{nm}$ fibers in terms of a simple solenoidal organization in 


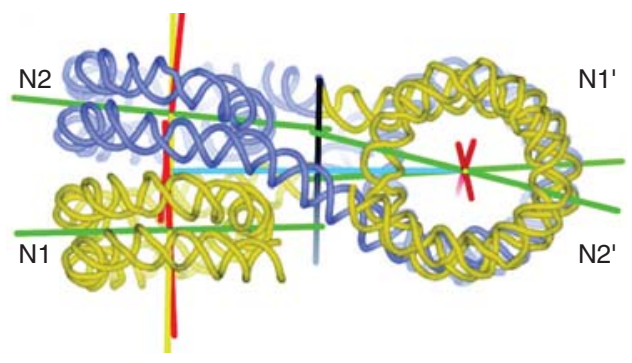

Figure 1. The DNA path of a tetranucleosome as determined by X-ray diffraction. The structure consists of two stacks of nucleosomes, with linker DNA passing back and forth between them. Thus, the primary interactions occur between alternate rather than adjacent nucleosomes along the DNA strand, creating a zigzag architecture. From Schlach et al. 2005.

which linker DNA followed the helical trajectory established in the nucleosome is probably incorrect, whereas subsequent suggestions involving some species of zigzag are probably closer to the mark.

\section{THE ROLE OF HISTONE H1}

In addition to the four "core" histones present as an octameric unit in the nucleosomes, a fifth histone, $\mathrm{H} 1$ or linker histone, is present in most nuclei. As its name implies linker histone is associated with linker DNA, and provides partial nuclease protection for $\sim 20 \mathrm{bp}$ of linker DNA. H1 contains a conserved globular region and extended amino- and carboxy-termini, the latter being rich in lysines and able to interact strongly with DNA. Although the exact location of $\mathrm{H} 1$ in chromatin remains controversial, it is clear that it plays a crucial role in promoting chromatin higher-order structure. H1-containing chromatin shows a distinct structural motif in which the entering and exiting linker DNA segments are brought together, perhaps promoting an overall zigzag arrangement (Bednar et al. 1998).

Arrays of nucleosomes depleted in $\mathrm{H} 1$ do not readily form $30-\mathrm{nm}$ fibers in vitro, and it is likely that loss of the charge neutralization effected by $\mathrm{H} 1$ is at least partly responsible. The crystals of tetranucleosomes discussed above were created without $\mathrm{H} 1$-and to date, no publications of the $\mathrm{X}$-ray structure of
H1-containing mono- or oligo-nucleosomes have appeared. Modest depletion of $\mathrm{H} 1$ from cells (achieved for the mouse by knocking out several of the six somatic $\mathrm{H} 1$ variants) results in reductions in nucleosome repeat length in some tissues whereas severe depletion is fatal for both mice and Drosophila (Fan et al. 2003; Lu et al. 2009). H1 depletion also inhibits the proper folding of chromosomes at mitosis (Maresca and Heald 2006). Interestingly, there is a strong linear relationship between $\mathrm{H} 1$ content and nucleosome repeat length in vivo (Woodcock et al. 2006), a feature that will act to maintain the electrostatic balance between DNA and histones.

\section{CHROMATIN FIBERS IN NUCLEI}

Thin sections of typical nuclei reveal a coarse differentiation between strongly staining heterochromatin (see the following for more on this term) adjacent to the nuclear periphery and nucleoli, and more weakly staining euchromatin dispersed throughout. Little internal structural detail can be resolved in either component. There are, however, a few special cases that are more informative. Echinoderm sperm nuclei contain uniformly distributed and highly compact chromatin, but on controlled reduction in ionic strength, the chromatin swells, revealing a mass of $\sim 30-\mathrm{nm}$ fibers. Although the fibers appear to be quite uniform in diameter, tomographic $3 \mathrm{D}$ reconstructions reveal an internal structure that consists of an irregular arrangement of nucleosomes and linker DNA but with a distinctive underlying zigzag motif (Horowitz et al. 1994). Sections of the transcriptionally inert chicken erythrocyte nucleus also reveal a uniform compaction of chromatin, which resolves into irregularly structured $30-\mathrm{nm}$ fibers when allowed to swell slightly by, for example, lowering the ionic strength. In contrast, 30-nm fibers are rarely seen in more typical nuclei from cycling cells, even after controlled swelling (Woodcock and Horowitz, 1995). These special cases in which the $\sim 30$-nm organization is revealed on swelling have several common features, including uniform core histone modifications, a greater 
abundance of linker histone (H1) relative to core histones, the presence of $\mathrm{H} 1$ variants with more positive charges and unusually long linker DNA. It is not clear why these properties lead to the appearance of distinct $\sim 30$-nm fibers in nucleo, but an attractive possibility is that these conditions favor intrafiber over interfiber interactions.

\section{NUCLEOSOME-NUCLEOSOME INTERACTIONS IN ISOLATED CHROMATIN FIBERS}

One approach to understanding chromatin fiber structure is to identify nucleosome-nucleosome interactions within the compact fiber. As noted, this cannot be achieved by direct EM observation of compact fibers, but ways to examine this have been devised. One study used the premise that the face-to-face nucleosome contacts that occur in crystals of mononucleosomes (Luger et al. 1997) would likely be seen in compact chromatin. The contacts involved the positively charged $\mathrm{N}$-terminus of histone $\mathrm{H} 4$ and the "acidic patch" that includes a portion of histone H2A. Dorigo et al. (2004) created $\mathrm{H} 4$ and $\mathrm{H} 2 \mathrm{~A}$ constructs with a cysteine in the putative contact region and reconstituted them into nucleosomal arrays. The arrays were then placed in conditions that promoted compaction and formation of S-S crosslinks between close cysteines. If the basic architecture of the array were solenoidal, crosslinking would result in a simple stack-of-coins conformation. In contrast, a zigzag arrangement would result in the creation of a ladder-like structure with the linker DNA segments forming the rungs. When cross-linking was initiated, the arrays clearly adopted the ladder-like structure, strongly supporting a zigzag architecture, albeit under somewhat artificial conditions. An approach that does not restrict internucleosome interactions to a specific histone-histone contact involves exposing nucleosomal arrays or whole cells to controlled formaldehyde crosslinking such that a few of the nucleosome-nucleosome contacts become covalently linked. Subsequently, the arrays are allowed to disperse in low salt and imaged by electron microscopy. With
Chromatin Higher-order Structure and Dynamics

his technique, termed EMANIC (EM-assisted nucleosome interaction capture) (Grigoryev et al. 2009), decondensed arrays appear in the open beads-on-a-string conformation (Fig. 2A), whereas crosslinked sites are seen as touching nucleosomes. Analysis of the crosslinking pattern of defined nucleosome arrays with $\mathrm{H} 1$ present once again reveals a predominantly zigzag pattern (Fig. 2 D, E). With $\mathrm{H} 1$ and $1 \mathrm{mM}$ $\mathrm{MgCl}_{2}$ together, some crosslinking is also seen between adjacent nucleosomes, suggesting these conditions favor a mixed, heteromorphic fiber architecture (Fig. 2F, G) in which the zigzag dominates, but is interrupted by the type of nucleosome-nucleosome interaction expected for a solenoidal architecture. Interestingly, computer modeling predicts heteromorphic fibers, and also shows that this arrangement allows greater compaction as it reduces the crowding of linker DNAs in the fiber interior (Grigoryev et al. 2009), and is more consistent with the higher packing ratios seen in compact chromatin (Daban 2000; 2003).

Although there is substantial support for a basic propensity of chromatin to form zigzag higher-order structures, results leading to alternate architectures have been presented. EM examination of long reconstituted nucleosomal arrays containing linker histone reveals remarkably uniform structures interpreted as consisting of solenoids in which adjacent gyres interdigitate (Robinson et al. 2006). This arrangement also increases the nucleosome packing density, and may thus better match the calculated in vivo packing. Additional studies of long nucleosomal arrays have led to the conclusion that the architecture is strongly influenced by linker length (Routh et al. 2008), increasing the potential complexity of chromatin structure in vivo, where varied linker DNA lengths are the norm.

\section{MECHANICAL PROPERTIES OF ISOLATED CHROMATIN FIBERS}

The development of sophisticated instruments using "optical tweezers" has made it possible to take an isolated chromatin fiber, anchor one end, and pull on the other end while 


\section{C.L. Woodcock and R.P. Ghosh}

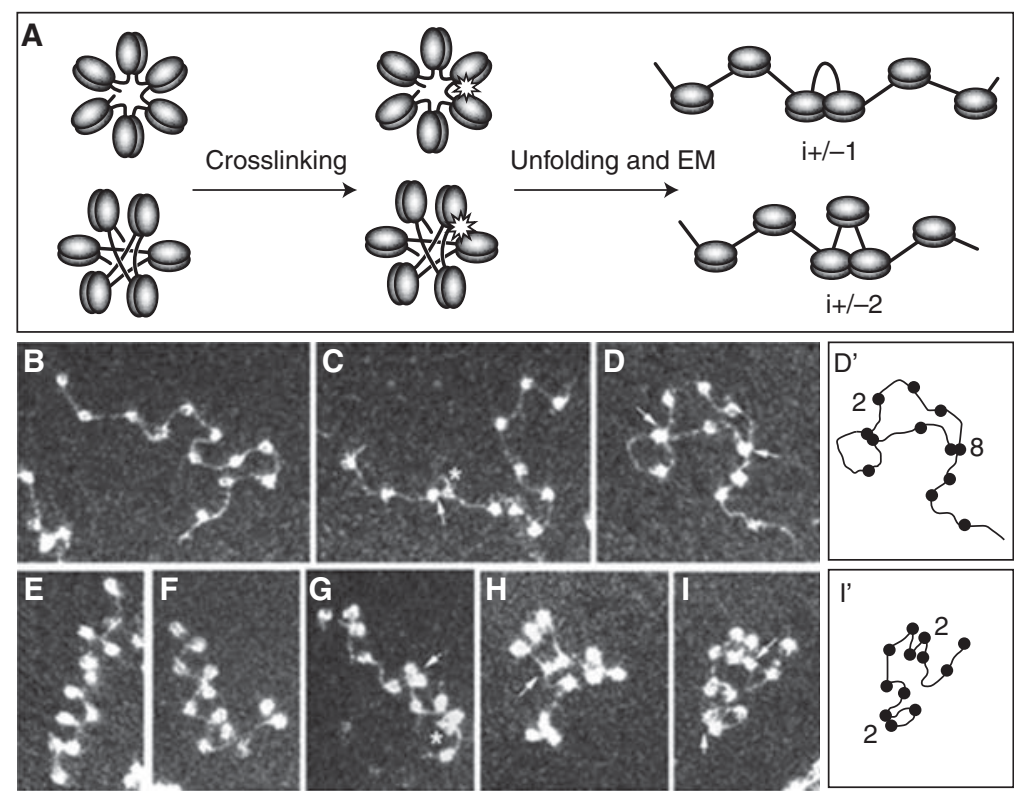

Figure 2. EMANIC analysis of internucleosomal interactions. (A) Scheme of the EMANIC procedure. The two models for the structure of the chromatin 30-nm fiber, namely solenoid (Upper) and zigzag (Lower), lead to dominant $\mathrm{i} \pm 1$ and $\mathrm{i} \pm 2$ internucleosome interactions, respectively. $(B-D)$ EM of nucleosome reconstitutes crosslinked in low salt without linker histone show few crosslinks. $(E-I)$ With H1 present, \pm 2 interactions predominate. $\mathrm{D}^{\prime}$ and $\mathrm{I}^{\prime}$ diagram the nucleosome arrays corresponding to the adjacent EM images. From Grigoryev et al. 2009.

measuring the force/length relationship. The applied force is expected first to break the weak internucleosomal bonds holding the fiber together, and ultimately, the much stronger intranucleosomal bonds. For a fiber with a zigzag arrangement, the breaking of individual nucleosome-nucleosome interactions is predicted to produce jumps in length with a consistent increment which would not be observed with solenoid type structures. Analysis of force/length curves for isolated chromatin fibers from chicken erythrocyte nuclei led to the conclusion that a zigzag arrangement predominated (Cui and Bustamante 2000). However, force/length data from a more recent study using reconstituted nucleosomal arrays (Kruithof et al. 2009) were interpreted as supporting a solenoidal architecture. The differences in starting material and ionic conditions used in these studies makes it difficult to evaluate the findings. In the future, studies encompassing a broader range of conditions and chromatin substrates should provide more definitive information from this potentially valuable technique.

\section{INSIGHTS FROM COMPUTER MODELING}

As noted earlier, computer modeling was consistent with the empirical finding of a heteromorphic fiber architecture. Current "mesoscale" modeling programs are supplied with sophisticated structural and chemical models of the nucleosome, H1 (if desired) and linker DNA, as well as the selected ionic environment (Arya and Schlick 2006). The changes in structure are then followed over a large number of small increments of time, and the final structures analyzed. As expected, the stochastic nature of the interactions involved lead to different final structures with each run of the program. However, consistent motifs emerge: the final structures are fibers, rather than spherical aggregates of nucleosomes, and, although zigzag 
arrangements of nucleosomes and linker DNA predominate, they may be interspersed with the bent linker motif characteristic of simple helical arrangements of nucleosomes. Modeling results are dependent on the accuracy of the input data, and computational limitations still preclude modeling at the atomic level. Nevertheless, this approach has the advantage of being able to sample a wide range of conditions, informing the selection of conditions for empirical examination with "real" chromatin. As computational speed continues to increase, modeling is likely to lead to more penetrating insights into chromatin architecture.

\section{LOCAL EVENTS THAT MAY MODULATE CHROMATIN FIBER ARCHITECTURE}

Much of recent work on chromatin secondary structure has featured fully defined nucleosomal arrays in which recombinant histones are assembled in vitro onto DNA containing strong nucleosome positioning sequences. Yet, as noted, even with highly uniform arrays, results have led to differing conclusions regarding the underlying architecture. In the nucleus, local effects of linker DNA length variability, core histone variants, and postsynthetic histone modifications are likely to contribute even more irregularity to secondary structures. Additional complexity is introduced by the presence of nonhistone proteins known collectively as Chromatin Architectural Proteins (CAPs) that influence chromatin conformation (Luger and Hansen 2005).

In addition to the "major" core histones, synthesized primarily in S phase and deposited at replication forks, there are numerous histone variants encoded by separate genes, which are often synthesized constitutively at low levels and incorporated differently. Some of these have been shown to play a critical role in establishing the local properties of chromatin in which they are embedded (Santenard and Torres-Padilla 2009). An important concept in this regard is the distinction between the major variant that is synthesized during $S$ phase and deposited as newly replicated chromatin, and minor variants. It has been proposed that the
Chromatin Higher-order Structure and Dynamics

incorporation of different $\mathrm{H} 3$ variants serves as a "bar code" (Hake and Allis 2006) contributing to the establishment of the local functional potential. Of the several variants of $\mathrm{H} 2 \mathrm{~A}, \mathrm{H} 2 \mathrm{~A} . \mathrm{Z}$ has been particularly well studied and shown to modulate chromatin higher-order structure. The X-ray structure of nucleosomes containing H2A.Z (Suto et al. 2000) reveals an enlargement of the 'acidic patch' at the nucleosome surface that appears to strengthen nucleosome-nucleosome interactions, and contribute to the highly compact chromatin surrounding centromeres (Greaves et al. 2007).

Another major source of chromatin variation is the large number of postsynthetic modifications of the core histones and $\mathrm{H} 1$ (Kouzarides 2007). They fall into two major categories, those that alter the charge on the histone, and those that establish binding sites for other proteins. Acetylation of lysines results in a reduction of the histone positive charge and weakens the DNA-histone interaction leading to a less compact chromatin secondary structure. The balance in activity between histone acetyl transferases (HATs) and histone deacetylases (HDACs) governs the acetylation status of a given region of chromatin. In general, hyperacetylation is the hallmark of active chromatin, whereas hypoacetylation is seen in repressed chromatin. A well-studied example is the acetylation of $\mathrm{H} 4$ at lysine 16. The loss of a positive charge at this site is likely to weaken the internucleosome interaction between the amino-terminus of $\mathrm{H} 4$ and the acidic patch on $\mathrm{H} 2 \mathrm{~A}$ discussed above. Indeed, the effect of this modification is to loosen the compaction of chromatin secondary structures (ShogrenKnaak et al. 2006; Fischle et al. 2003). An example of a modification that establishes a binding site is the methylation of $\mathrm{H} 3$ at lysine 9. The modification creates a binding site for a domain of the HP1 protein (Jacobs and Khorasanizadeh 2002). As discussed below, HP1 binding is associated with the formation and propagation of compact heterochromatin. Work on postsynthetic histone modifications has led to the widely discussed concept that, collectively, they constitute a "histone code" that defines the local structural and functional potential 
of a region of chromatin (Jenuwein and Allis 2001).

Chromatin architectural proteins, as their name implies, do impact chromatin structure, mostly by increasing the compaction state (Luger and Hansen 2005). This appears to be their sole common feature, because otherwise they are highly diverse in both structure and in chromatin binding mechanism. Binding may be restricted to specific DNA sequences (Polycomb), methylated DNA (MeCP2), or specific histone modifications (HP1). Only in a few cases, such as the SIR family of DNA-binding regulatory proteins is a detailed account of their molecular interactions with chromatin and with each other emerging (Buchberger et al. 2008). Some CAPs contain more than one DNA/chromatin binding sites, allowing them to act as inter- and/or intrafiber bridges, and thus promote chromatin compaction.

Chromatin remodeling complexes may be considered as a distinct class of CAPs. Several families of these large multicomponent complexes have been identified. In vitro, they have the ability to restructure, eject, or move nucleosomes in an ATP-dependent manner (Clapier and Cairns 2009). This type of activity in vivo will likely influence chromatin secondary structure, and, by altering the local positioning of nucleosomes, modulate chromatin folding. An interesting case has been reported of the combined effect of a histone variant (H2A.Z), a histone modification $(\mathrm{H} 3 \mathrm{~K} 9 \mathrm{Me})$, and a CAP (HP1), all of which act to increase chromatin compaction (Fan et al. 2004). Thus, the proposed histone code based on histone modifications may need to be expanded to include synergistic effects of histone variants. A novel function in maintaining the pluripotential "open" state of chromatin for the remodeling factor Chd1 (Gaspar-Maia et al. 2009) may presage new insights into the roles of these complexes.

A scenario of chromatin secondary structure that, in our opinion, appears to fit all the current data involves chains of nucleosomes that are sufficiently close packed in vivo to interdigitate, effectively merging with each other and preventing individual fibers being seen by microscopy. Nevertheless, the overall trajectory of a chain of nucleosomes traces out an irregular $\sim 30$-nm fiber within the nucleus. Fragmentation of chromatin by limited nuclease digestion followed by elution from nuclei results in individual fibers becoming separated, and available for microscopic and biochemical characterization. Irregularity dominates the architecture of isolated native chromatin fibers, reflecting the heterogeneity in linker DNA length, and composition of histone variants, histone postsynthetic modification, and CAPs. The end result is likely a hybrid (heteromorphic) organization with a predominantly zigzag architecture interspersed with other structural motifs. Most of these sources of irregularity are dynamic, changing at different time scales. Thus, it is probably futile to expect that chromatin secondary structure can be defined with the precision expected from studies of other biological fibers such as microtubules or Tobacco Mosaic Virus. Thus, seeking a defined secondary structure for chromatin may indeed be "Chasing a Mirage" (van Holde and Zlatanova 1995).

Structural Order Beyond the 30-nm FiberElectron Microscopy Results

The lack of definitive information on chromatin secondary structure complicates the question of whether there is a bona fide set of hierarchical structures above the 30-nm fiber. Although there is no doubt that some higher levels of organization are present- the metaphase chromosome being an obvious example-precisely what form they take and whether they are truly hierarchical is still a matter of intensive study and debate. Much of this work uses the light microscope and has been limited in resolution to $\sim 250 \mathrm{~nm}$ by the properties of light. The impact of a $\sim 250$-nm resolution limit is well illustrated in a study that considers the possible structural arrangement of large genetic loci $(\sim 400 \mathrm{~kb})$ by matching the predictions of a number of models for large-scale chromatin structures with actual observations (Muller et al. 2004). Somewhat surprisingly, at the resolution attainable with a high quality confocal 
microscope, all models fitted the data equally well! The higher resolution of the EM, coupled with tomography, and applied to thin sections has revealed short regions of fiber-like structures with a variety of diameters, none particularly consonant with a hierarchical series based on a 30-nm organization (Konig et al. 2007; Belmont et al. 1989, 1994).

More recently, however, significant improvements in technique have been made using an in vivo immuno-gold technique that promises to lead to important insights (Kireev et al. 2008). The introduction by Belmont and colleagues of techniques for constructing cell lines containing large foreign DNA segments that are "self identifying" through an ingenious use of the lac operator/repressor system (Belmont et al. 1999) has had a major impact within the field and is widely used. A recent adaptation of the method involving the intracellular injection of antibodies specific for the introduced chromatin followed by gold decoration of the antibodies, thin sectioning and EM observation reveals clear-cut cylindrical fibers as can be seen in the stereo pair in Figure 3 (Kireev et al. 2008). The fibers ranged in diameter from $120 \mathrm{~nm}$ to $170 \mathrm{~nm}$ with different mean values in two cell types examined. Only rarely were individual 30-nm-like fibers seen, and, as the authors note, it remains to be seen whether the thick fibers constitute one level in a hierarchical folding series. Hopefully, further exploitation of the technique will lead to long-awaited progress in our understanding of chromatin organization in situ.

Structural Order Beyond the 30-nm fiberLight Microscopy Results

The ability to identify specific segments of chromatin using light microscopy has provided important insights into interphase chromatin organization. However, in evaluating the impressive body of information obtained with the light microscope, it is important to bear in mind the preparative method used in each case, because this may significantly modulate the outcome of an investigation, especially when close to the resolution limit. Studies
Chromatin Higher-order Structure and Dynamics

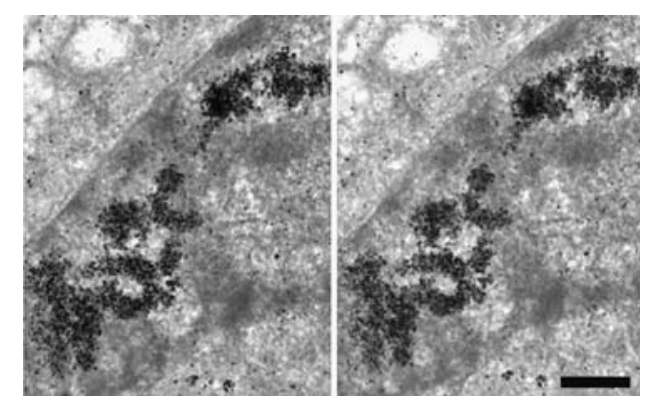

Figure 3. Stereo pair of a section of nucleus in which a large region of chromosome is decorated with gold particles. Clear fiber-like structures of the order of $100 \mathrm{~nm}$ are seen. Scale is $500 \mathrm{~nm}$. From Kireeva et al., 2008.

with living cells are likely to provide the most reliable data, whereas the use of fluorescence in situ hybridization (FISH) requires a denaturation step which may introduce structural perturbations.

Muller et al. (2004) reported that $\sim 400 \mathrm{~kb}$ segments of nontranscribed chromatin occupy roughly spherical volumes with a diameter not much greater than the resolution limit of the microscope. A $\sim 400 \mathrm{~kb}$ length of DNA translates into $\sim 2000$ nucleosomes, and, if present as a linear $\sim 30 \mathrm{~nm}$ fiber with 10 nucleosomes per $10 \mathrm{~nm}$ length would have a length of $\sim 2$ $\mu \mathrm{m}$. Thus, in this case, an untranscribed region of chromatin must be extremely tightly packed. However, an underlying fiber-like organization below the resolution of the light microscope cannot be excluded (Tumbar et al. 1999). It will be very interesting to see if the higher resolution offered by subdiffraction light microscopy will reveal any internal structure in these compact regions. The situation changes dramatically on transcription, when loci typically expand, often becoming linear and adopting a beaded form. In an informative study, Shopland et al. (2006) examined the intranuclear distribution of a $4 \mathrm{Mb}$ segment of mouse chromosome 14 that included four gene clusters separated by gene "deserts." By labeling the gene clusters and deserts with different colored fluorescent probes, it was possible to trace the conformation of this large segment of chromatin (Fig. 4). Four patterns occurred in roughly 
C.L. Woodcock and R.P. Ghosh

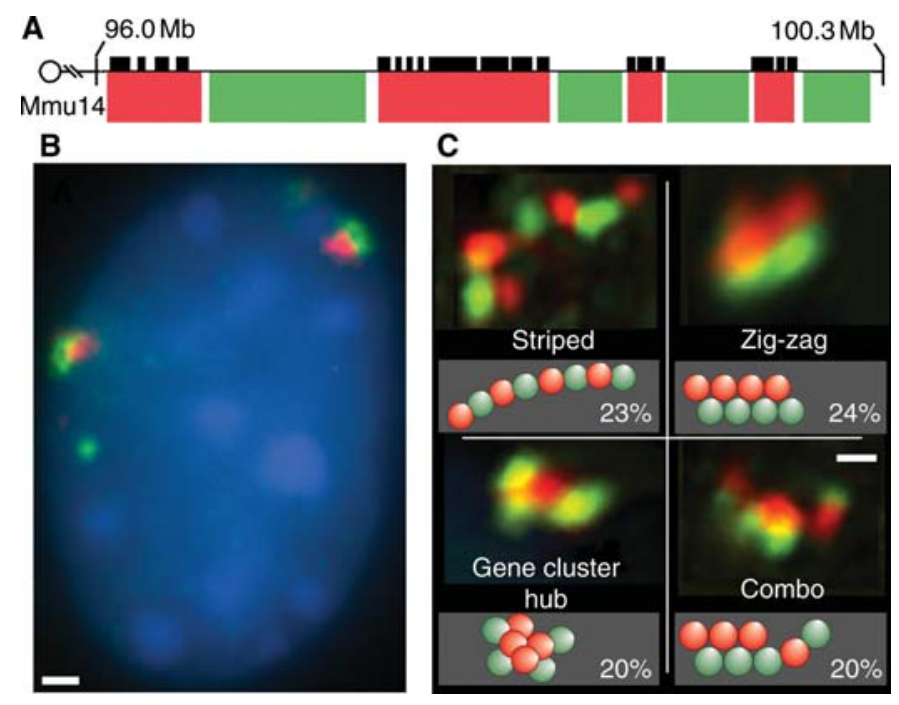

Figure 4. (A) A large locus consisting of 4-Mbp region containing regions of gene "deserts" (red fluorescence) and gene clusters (green fluorescence) is seen in the nucleus in multiple configurations $(C)$. In general, gene deserts are more closely associated with the heterochromatin at the nuclear periphery $(B)$. Scale is $1 \mu \mathrm{m}$. From Shopland et al. 2006.

equal proportions, ranging from linear "striped" arrangements to more compact clusters. Interestingly, the inactive segments were often clustered and oriented closer to the nuclear periphery than the gene-rich segments, in keeping with the preferential location of inactive chromatin in peripheral heterochromatin.

\section{HETEROCHROMATIN AND EUCHROMATIN}

The terms heterochromatin and euchromatin refer to states of compaction and transcriptional potential rather than categories of chromatin higher-order structure per se. Nevertheless, they are used ubiquitously in the literature and merit a brief mention. Heterochromatin was originally defined well before the discovery of DNA as regions of nuclei that stained strongly with basic dyes (Heitz, 1928) and, together with its counterpart euchromatin, has provided a useful qualitative indication of chromatin compaction state. In general, heterochromatin tends to be located at the nuclear periphery, where specific interactions with the envelope may occur and often forms blocks surrounding the nucleolus. These distinctions can be clearly seen in light and electron micrographs. Transcription is largely confined to euchromatin, and it is interesting to note that Heitz presciently suggested a functional difference between the two forms.

Today, the term heterochromatin is more loosely applied, and is often extended to include transcriptionally silent regions of chromatin regardless of their staining properties. An important distinction is made between constitutive and facultative heterochromatin. Constitutive heterochromatin is always compact, and tends to be enriched in repetitive, gene-poor, and late replicating DNA sequences, whereas facultative heterochromatin can reversibly undergo transitions from a compact, transcriptionally inactive state to become more open, and transcriptionally competent. In a recent review, Trojer and Reinberg (2007) suggested that facultative heterochromatin be molecularly defined as condensed, transcriptionally silent chromatin regions that decondense and allow transcription within temporal, spatial, or parental/heritable contexts. During embryogenesis, for example, the amount of facultative heterochromatin increases as unwanted sets of genes are progressively shut down until at maturity, a cell expresses only the genes appropriate for that tissue. The reverse occurs when, 
for example, differentiated cells are reprogrammed to become stem cells. These events are typically accompanied by profound changes in histone variants, histone modifications, and the presence of CAPS. An important feature of heterochromatin is its propensity to "spread" to adjacent regions of chromatin (Liaw and Lustig 2006; Hines et al. 2009).

A recent comparison of properties of heterochromatin and euchromatin as diffusion barriers has yielded interesting and provocative results (Bancaud et al. 2009). Measurements of diffusion constants of large polymers within these nuclear compartments confirmed that heterochromatin constitutes a more crowded environment, leading to the more efficient trapping of chromatin binding proteins such as histone $\mathrm{H} 1$. The crowding effect was also suggested to assist in the maintenance of heterochromatin. Further, kinetic analyses indicated an anomalous component of diffusion that was interpreted in terms of a fractal chromatin organization at spatial scales below $100 \mathrm{~nm}$. This indicates a physical arrangement more similar to the "molten globule" state suggested by the lack of structure in cryo sections than to the classic $30-\mathrm{nm}$ fiber.

The ubiquitous location of heterochromatin at the nuclear periphery and association with the nuclear lamina and nuclear envelope suggests that this location is both structurally and functionally important. It was therefore surprising to read that, in the rod photoreceptor cells of nocturnal, but not diurnal, mammals, heterochromatin is concentrated in the center of nuclei (Solovei et al. 2009). The authors postulate that with this arrangement, nuclei act as collecting lenses, providing additional sensitivity in very low light environments. This unexpected finding underscores the plasticity of chromatin organization.

\section{CHROMATIN LOOPS}

The concept of large scale chromatin loops is important in many scenarios of chromatin function. The generic term "chromatin loop" is used for a variety of phenomena that play important roles in nuclear organization and
Chromatin Higher-order Structure and Dynamics

function, but otherwise may have little in common. Further, it is not at all clear whether the various loop phenomena that have been reported constitute distinct level(s) of chromatin higher-order structure (Kadauke and Blobel 2009). It is clear, however, that loop phenomena vary in terms of their stability. For example, enhancer-promoter loops that facilitate transcription are transitory, dynamic events, whereas other types of loops appear to be more stable. An early observation that has stood the test of time is the formation of a DNA "halo" around isolated nuclei exposed to a mild treatment that releases histones (Cook and Brazell 1975). The halos were shown to consist of supercoiled DNA, suggesting that the DNA twisting that occurs when histones are removed from nucleosomes is preserved by being anchored in the residual nuclear structure. This finding is also consistent with the concept of an insoluble proteinaceous nuclear matrix or karyoskeleton to which chromatin loops are anchored by DNA sequences referred to as matrix attachment regions (MARs). However, because it has not been possible to define its composition and structure, the matrix remains a useful working concept rather than a well-accepted structure in the same sense as the cytoskeleton (Pederson 2000). A somewhat different perspective involves the "looping out" from chromosome territories of large segments of genome that are related in terms of transcriptional activity.

The supposition that these different loop phenomena reflect the same underlying chromatin organization was called into question by a surprising recent finding that halo diameter was related to the spacing of origins of replication during the previous $S$ phase (Courbet et al. 2008). Under conditions of rapid replication, origins were widely spaced, leading to large halos, whereas slow replication triggered the firing of additional replication origins, and led to smaller halos. Halos can also be generated from metaphase chromosomes, and giant meiotic lampbrush chromosomes provide a particularly compelling example of large chromatin loops and their relation to transcriptionally coupled genes. Large-scale chromatin loops are featured in some models of chromatin and 
chromosome organization, but how they are integrated into chromatin/nuclear structure and whether they constitute a distinct hierarchical level of chromatin organization remains to be established.

The interaction between enhancers and promoters probably represents an unrelated manifestation of chromatin looping that is critical for transcriptional activity. Enhancer sequences are often many $\mathrm{Kb}$ distant from promoters, and may be located upstream, downstream, or on a different chromosome. There seems now to be a consensus that physical interaction between enhancer and promoter is necessary to initiate transcription (Visel et al. 2009), but how widely separated loci are brought into contact has remained an open question. One possibility, supported by recent data (Nolis et al. 2009) is that sequence-specific transcription factors bind to both enhancer and promoter, and also bind strongly with each other. In support of this hypothesis, "decoy" transcription factor binding sites placed between a promoter and its enhancer blocked transcription, suggesting that they trapped the enhancer in unproductive loops (Nolis et al. 2009).

Finally, the recently introduced technique of chromosome conformation capture (3C) and related methods, which allow mapping of physical chromatin interactions in vivo, is providing growing evidence for physical interactions between distant loci other than enhancerpromoter juxtapositions (Gondor and Ohlssen 2009). Hints that there must be mechanisms for bringing specific loci together has come from the common occurrence of some chromosomal translocations, especially those leading to human diseases. For example, Roix et al. (2003) showed that genes associated with chromosome translocations leading to human lymphomas tend to be in physical proximity and located toward the nuclear interior. The data also suggested that the phenomenon was dependent on the "higherorder spatial organization" of the genome rather than the sequences of the genes involved. Human prostate cancer offers a system for tracking the physical proximity of two loci. The TMPRSS and ERG genes are $\sim 3 \mathrm{MB}$ apart on human chromosome 21 , but become fused in $\sim 50 \%$ of prostate cancer cases, apparently in response to hormone levels. Using FISH to follow the locations of the two genes, Mani et al. (2009) showed that, upon hormone treatment, the two genes became physically close in a significant proportion of cells. The mechanism(s) involved in this large-scale motion remain unknown. Another recent study of intranuclear chromatin associations capitalizing on massively-parallel sequencing has yielded important insights into the large scale organization of chromatin (LiebermanAiden et al. 2009). Although the technique in its current form is limited to megabase scale resolution, the data clearly indicated that active and inactive chromatin occupy different domains within chromosome territories. Further, at this level of resolution, chromatin conformation was consistent with a knot-free, fractal globule organization. In the representation of their conclusions, Lieberman-Aiden et al. show modeled nuclei with chromatin modeled as worm-like structures of uniform diameter, and it is important to appreciate that these structures are not intended to correspond to a specific level of chromatin higher-order structure. It is interesting to note that whereas this study indicated a fractal-like organization at very large scales, unrelated work on the accessibility of differentially packed chromatin suggested a fractal conformation for chromatin below $10 \mathrm{~nm}$ (Bancaud et al. 2009). Hopefully, these two sets of findings obtained through widely divergent experimental approaches, augur a future in which chromatin organization can be studied effectively at many different levels. The study of chromatin long range organization using $3 \mathrm{C}$ and related techniques (Rusk, 2009) is clearly at an early stage in development, and promises to provide important insights into the mechanisms that bring distant loci together and their relationship to chromosome territories and chromatin dynamics.

\section{The Metaphase Chromosome}

The metaphase chromosome, in which the DNA is compacted some 10,000 - to 20,000 fold, is the one consistent manifestation of chromatin higher-order structure. It is therefore sobering to reflect that despite a great deal of 
research, there is still no widely accepted model for the internal organization of this critical cellular component. Andrew Belmont, who has contributed seminal work in the field, has aptly applied the quotation "a riddle, wrapped in a mystery, inside an enigma" to illustrate the complexities of metaphase chromosome structure. There are several features that must be accounted for in any viable model. For a given species, the diameter of chromosome arms at metaphase is quite constant, although there are wide variations in chromosome diameter and number from species to species. The width constancy appears to be independent of DNA sequence. This is seen clearly in cases in which a chromosome bears a large insert, either as a response of the cell to a genetic defect that is overcome by accumulating multiple tandem repeats of the mutated gene (e.g., Sullivan and Bickmore 2000), or by insertion of large tandemly repeated loci. Typically, these inserts, which appear at metaphase as homogeneously staining regions (HSRs) on account of their lack of band/interband organization, have a uniform diameter that closely matches that of the rest of the chromosomes. This indicates that something other than DNA sequence is establishing the underlying folding pattern that governs chromosome diameter.

It is well-established that, with the exception of polytene systems, chromosomes are unineme, in that a single strand of DNA, albeit multiply folded, extends from one end (telomere) to the other (reviewed in Gall 1981). Although for a particular chromosome, metaphase banding patterns and gene locations are consistent from cell to cell, the spatial resolution of light microscopy provides very limited structural information in the context of a chromosome arm that may have a diameter of $\sim 500 \mathrm{~nm}$. There is, however, a great deal of information relevant to the overall architecture of metaphase chromosomes.

An early and striking result was derived from treating chromosomes to remove histones, which, like similar treatments of nuclei, revealed a "halo" of DNA loops surrounding a denser core with a size and shape comparable to the starting chromosome (Earnshaw and Laemmli
Chromatin Higher-order Structure and Dynamics

1983). The core structures were termed "scaffolds" and it was postulated that they formed the structural basis of chromosome architecture. DNase treatment could be used to isolate the scaffolds, which contained specific DNA sequences (scaffold-attachment regions or SARS) and were enriched for a few proteins thought to be essential for scaffold, and hence chromosome structural integrity. Avery fruitful approach to chromosome structure that allows experimental manipulation uses amphibian egg extracts, which can be manipulated to recapitulate, in sperm nuclei, the essential cell cycle events of DNA replication, chromosome formation and mitosis (Lohka and Masui 1983). Importantly, the system allows the roles of individual components to be explored by their addition to or removal from the extract. Support for the scaffold hypothesis was provided by the observation that, in chromosomes formed in vitro in egg extracts, some components isolated from scaffolds were distributed axially at metaphase rather than uniformly. However, subsequent experiments established that the situation was more complex. For example, topoisomerase II could be removed from chromosomes assembled in vitro without compromising their structure (Hirano and Mitchison 1993), but was essential for proper chromosome assembly and separation of sister chromatids at anaphase. Further, genetic screens for chromosome segregation mutants revealed a family of "structural maintenance of chromosomes" (SMC) proteins (Hirano 2006). Also identified were two complexes, cohesin and condensin that contain SMCs and other proteins and, in addition to other functions, appear to be involved in sister chromatin adhesion and chromosome condensation (Hagstrom and Meyer 2003). Condensin is consistently seen to have an axial distribution in chromosomes, and in its absence or mutation, proper condensation fails.

A completely different approach, in which micromechanical properties of chromosomes are measured, has introduced a dramatically different aspect of their structure. Importantly, these results have had the salutatory effect of requiring fresh thinking to reconcile the seemingly disparate sets of data. The strategy is to 


\section{C.L. Woodcock and R.P. Ghosh}

isolate chromosomes either from mitotic cells or egg extracts, hold each end with micropipettes, pull on one end, and measure the force needed for a given length increase (Houchmandzadeh et al. 1997; Poirier and Marko 2003). The environment of the chromosome can be altered by modulating the buffer with cations etc., and the effects of nucleases or proteases can be determined. The results show that chromosomes exhibit a remarkable degree of elasticity (Fig. 5), repeatedly returning to the original length after being stretched $\sim$ fivefold. The general conclusion from several studies is that mitotic chromosomes consists of a network or gel in which individual chromatin fibers are connected by crosslinking elements, presumably proteins (Fig. 6). It was also possible to estimate a mean distance between crosslinks of $\sim 15 \mathrm{Kbp}$ by applying restriction enzymes with different cutting frequencies to chromosomes before stretching (Poirier and Marko, 2002). The estimated distance between crosslinks is close to that calculated for the chromatin loops associated with chromosome scaffolds (Gasser et al. 1986). One surprising feature of the work was the large difference in flexibility between "native" chromosomes extracted from cells by micromanipulation and those assembled in egg extracts in vitro. The latter were almost two orders of magnitude more flexible, suggesting that their non-DNA components were thin, rigid, and highly extensible (Houchmandzadeh et al. 1997)

In its simplest form, the network model seems to be consistent with the lack of consistent large-scale structures seen in cryo-sections of HeLa cell chromosomes (Eltsov et al. 2008) or in EM tomograms of chromosomes formed in Xenopus egg extracts (König et al. 2007), but at odds with hierarchical models of chromosome structure, which would predict that stretching a chromosome would result in the unfolding of hierarchical levels with stepwise reductions in chromosome diameter. The network model also seems to contradict the many light microscopic observations of an axial localization of key proteins, especially condensins known to be involved in chromosome compaction.
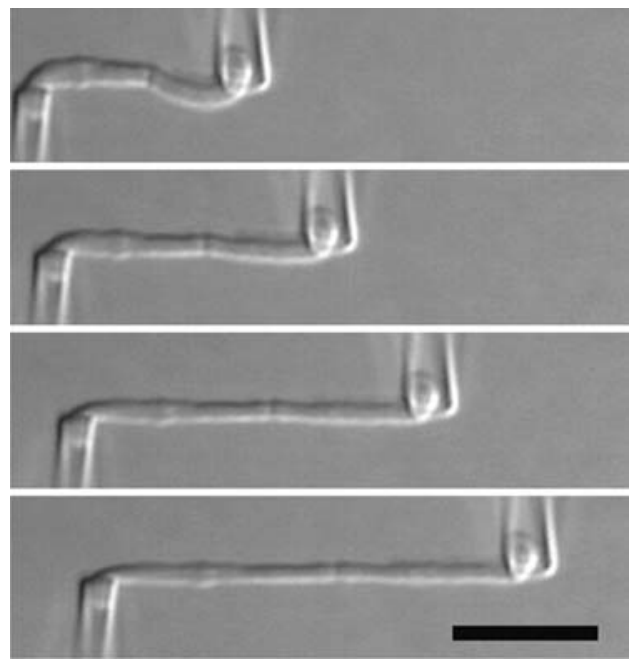

Figure 5. Time sequence showing the extension of a metaphase chromosome in which the two ends are anchored by micropipettes. The diameter remains quite constant throughout. From Marko 2008.

Recently, however, models that account for most of the apparently disparate observations have been put forward. An architecture based on observations of early chromosome condensation that involves hierarchical folding with an axial "glue" provided by condensin (Fig. 7) has been proposed by Kireeva et al. (2004), and a more elaborate system with specific locations and roles for topoisomerase II, cohesin and condensin has been put forward by Marko (2007). In the case of the model in Figure 7, a delicate balance between the forces maintaining the $200-250 \mathrm{~nm}$ and $500-750 \mathrm{~nm}$ fibers would

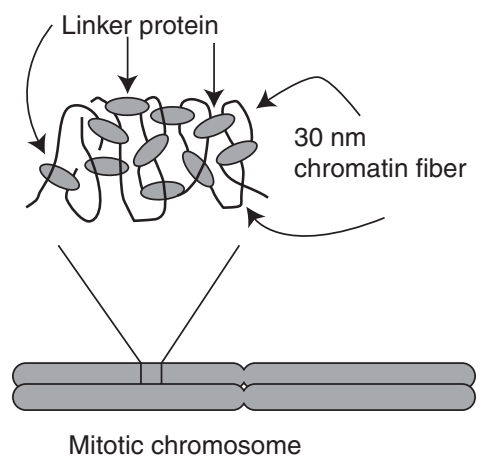

Figure 6. Extensible net model of mitotic chromosome structure derived from force-extension measurements. From Poirier and Marko 2002. 


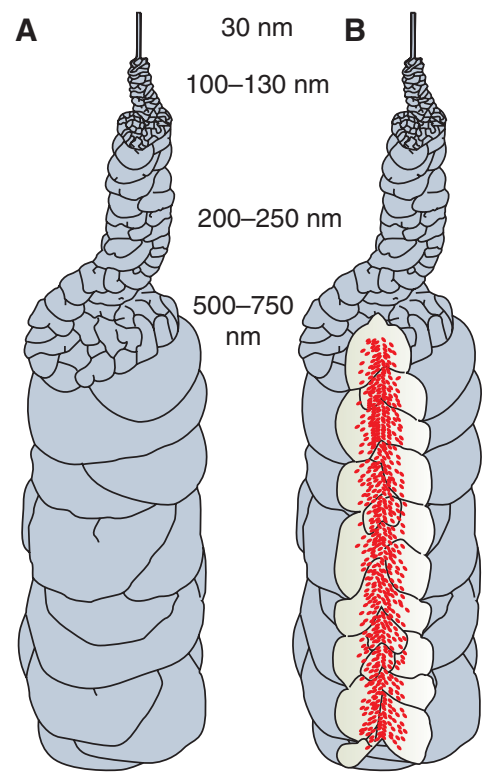

Figure 7. Model of chromosome formation that incorporates the concept of a central axis enriched in condensins (red dots) and irregularly folded chromatin fibers. From Kireeva et al. 2004.

be needed to produce the stretching effect shown in Figure 5. As noted by Belmont (2006), many questions remain unsolved, and there are probably additional proteins, levels of complexity, and redundant mechanisms to be discovered before the complete story of chromosome architecture, formation and maintenance is uncovered. Indeed, a new activity, RCA (regulator of chromosome architecture), that can condense chromosomes in the absence of condensins has recently been proposed (Vagnarelli et al. 2006). To date, no satisfactory explanation of the constant diameter of chromosomes has emerged. Thus, the simple-appearing metaphase chromosome is far from simple, and fully understanding its composition, formation, maintenance and architecture will likely remain a challenge for some time.

\section{LAMPBRUSH AND POLYTENE CHROMOSOMES}

These two special and quite rare manifestations of chromatin provide some insight into the
Chromatin Higher-order Structure and Dynamics

surprisingly wide range of nuclear organizations that "work." Lampbrush chromosomes (LBCs) are largely confined to meiosis in oocytes, especially avian and amphibian, but are also present in the large unicellular alga Acetabularia. They appear to be an adaptation for maximizing transcriptional output to serve thousands of cells in the developing embryo and the macroscopic cell body in Acetabularia. Light microscopy reveals an axial core from which loops of varying size extend. Loops contain transcription units that can be seen in the electron microscope to be loaded with RNA polymerases and continuously transcribing. Although the loops are the most conspicuous features of LBCs, they represent only a small fraction of the genome, the bulk forming the compact chromatin of the axial cores. The fact that sperm nuclei exposed to extract from oocytes at the lampbrush state develop into haploid LBCs (Gall and Murphey 1998) indicates that the signals needed to transform a chromosome into the lampbrush state evidently reside in the oocyte. A key question is whether this loop organization is unique to lampbrush chromosomes or whether it offers an especially clear example of a general organizing principle for transcriptionally active chromatin. Although the growing evidence for the ubiquitous organization of chromatin into large scale loops containing functionally related genes is consistent with the latter interpretation, there is also compelling evidence transcription of "normal" chromatin is organized quite differently (Hu et al. 2009).

Polytene chromosomes are formed by repeated mitoses without nuclear division and consist of genomes precisely aligned in trans. They are especially prominent in dipteran larva, with 1024 copies of the genome being present in the third instar of Drosophila. In the light microscope, a unique pattern of bands and interbands is seen, allowing the locations of individual genes to be mapped cytologically. It has long been recognized that at the sites of transcriptionally active genes, "puffs" appear as the chromatin is locally decondensed. Disappointingly, polytene chromosomes have not yielded much insights into chromatin organization in general. 
In the electron microscope, their alignment appears far from regular, and it is as difficult to recognize and follow individual chromatin fibers as in normal diploid nuclei. Polytene chromosomes may also be used to infer the structural organization of interphase chromatin. For example, a component that, when depleted or mutated, results in disorganization of polytene chromosomes, may be involved in sister chromatid adhesion. Polyteny is also seen in the first stages of the development of the nurse cells surrounding the Drosophila ovary. As nurse cells mature, the polytene alignment breaks down, and the nuclei become polyploid. Surprisingly, in flies with mutations of putative condensin 2 components, the polytene chromosomes fail to disperse at the appropriate time, whereas the same mutations induce dispersion of salivary gland chromosomes (Hartl et al. 2008). These findings may lead to further insights into the complex events controlling polytene chromosome integrity.

Both lampbrush and polytene chromosomes dispel the idea that transcription is obligatorily confined to "factories" that contain RNA polymerases and the multiple other factors necessary for RNA synthesis and processing (Cook 1999). Observation of lampbrush loops clearly indicates that it is the polymerases that move along the loops rather than vice versa. Similarly, it seems very unlikely that DNA replication in polytene chromosomes occurs at replication factories through which DNA is translated (Cook 1999). These considerations do not lessen the potential importance of transcription and replication factories in general, but again emphasize the remarkable plasticity of nuclear organization.

\section{CHROMATIN DYNAMICS}

The profound structural rearrangements that chromatin must undergo during the cell cycle underscores one aspect of the dynamic nature of chromatin. There is, in addition, compelling evidence of an important role for local structural changes in compaction and/or position of specific genetic loci likely to be of critical importance in their transcriptional regulation.
In fact, most of the functionally important characteristics of chromatin show dynamic behavior in the sense of time-dependent changes. These include the mobility of many chromatinbinding components, including chromatin architectural proteins (Phair and Misteli, 2000), and the status of posttranslational modifications, especially of the core histones. Many of these phenomena are likely to impact local and global chromatin conformation and thus modulate higher-order structure.

The study of chromatin dynamics in all of its manifestations has opened up exciting new perspectives-indeed, a literature search reveals over 60 reviews with titles containing the terms "chromatin" and "dynamics" (e.g., Huebner and Spector 2010). Here, we focus on aspects of dynamics that are likely to impact higher-order structures. All involve changes in location and/ or shape of specific genetic loci that are thought to have important functional implications.

As the field of chromatin dynamics has developed, it has become increasingly clear that a number of underlying physical and molecular phenomena are involved, and that to understand them fully, information at many different levels is required. For a given locus these may include the temporal and spatial scales of observed movements, their energy dependence, and their location, both within the nucleus and within their specific chromosome territory. One important goal of current research is to be able to relate these changes in location/shape to the underlying physical changes in chromatin higher-order structure. For movements that are correlated with changes in transcriptional activity, it is also important to determine whether a change in location is a prerequisite for, or a consequence of, the altered level of RNA synthesis.

Advances in light microscopy techniques over the past $\sim 25$ years have allowed chromatin dynamics to be examined with steadily improving temporal and spatial resolution, and has enabled three principal types of dynamic change to be recognized (Soutoglou and Misteli 2007). These differ in the extent of motion, their energy dependence, and the time scales involved (Fig. 8). Early studies tracking changes in 
location of large segments of chromatin and whole chromosome territories indicated that, when corrected for nuclear rotation, the regions were essentially immobile over distances $>0.4$ $\mu \mathrm{m}$ (Cremer et al. 1982, Diboni and Mintz 1986, Shelby et al. 1996). However, more recent work combining higher spatial resolution with in vivo labeling of smaller defined segments of chromatin, revealed a more complex scenario with different regions of chromatin showing very different mobilities. For example, a segment of budding yeast chromatin located near a centromere was found to show constrained random walk diffusive motion with a confinement radius of $\sim 0.3 \mu \mathrm{m}$ (Marshall et al. 1997), that was independent of the metabolic state of the cell. The rate of mobility of this region of chromatin was calculated to be approximately three orders of magnitude lower than expected for free DNA of similar length (Marshall et al. 1997), leading to the suggestion that the locus was tethered within the nucleus, perhaps to the nuclear envelope or some internal structure. A detailed study of chromatin
Chromatin Higher-order Structure and Dynamics

mobility in Drosophila spermatocytes using loci labeled with the lac repressor system recorded relatively large movements during short time intervals in early nuclei. Movements of this magnitude would be expected to result in displacements of over $4 \mu \mathrm{m}$ in $1 \mathrm{~h}$ and cover the entire 11-12 $\mu \mathrm{m}$ diameter of a nucleus in $6-7 \mathrm{~h}$ (Vazquez et al. 2001). However, when long time periods were examined, it was clear that loci were constrained within a volume approximately equivalent to a chromosome territory. Another important finding was that spermatocytes in late G2 stage and approaching meiosis showed greatly reduced chromatin mobility, suggesting that some form of additional tethering precedes chromosome condensation.

A comparison of the mobility of different chromosomal sites in yeast also revealed striking locus- and cell cycle-dependent differences in chromatin dynamics (Heun et al. 2001). Some chromatin regions showed occasional large $(\sim 0.5 \mu \mathrm{m})$ movements over time periods as short as 10 seconds that were inhibited in ATPdepleted cells and thus dependent on the
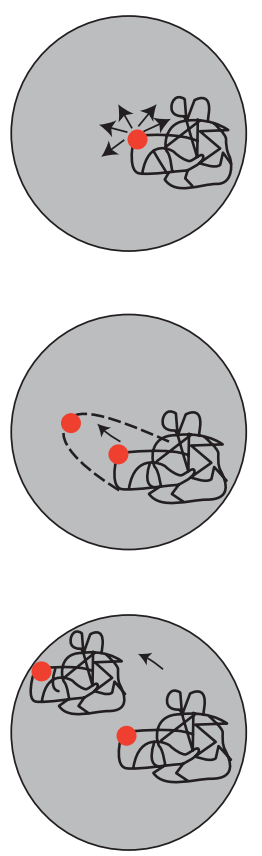

Short-range motion

-Locally constrainemd within $\sim 1 \mu \mathrm{m}$

-Fast (seconds)

-Random direction

-ATP dependent

-Frequent

-Occurs by default

Long-range motion

-Several micrometers

-Slow (minutes)

-Directed

-ATP/motor dependent

-Rare

-In response to physiological cues (cell cycle)

Genome Reorganization

-Several micrometers

-Slow (hours, days)

-Global changes

-Likely requires cell division

-In response to physiological cues

(differentiation, development)

Figure 8. The different forms of large-scale motion that contribute to chromatin dynamics. From Soutoglou and Misteli 2007. 
metabolic state of the cell. The fact that the magnitude of these large movements is similar for organisms with widely different nuclear volumes has important structural and functional implications. For example, in budding yeast, which has a nuclear diameter of $\sim 2 \mu \mathrm{m}$, movement within a radius of $\sim 0.5 \mu \mathrm{m}$ would allow access to a large portion of the nuclear volume. In contrast, in a typical mammalian nucleus of $\sim 10 \mu \mathrm{m}$ in diameter this motion would explore only a thousandth of the nuclear volume. In yeast, the effectively large displacements of loci within nuclei may promote the observed high recombination frequency, which may require substantial intermingling among different regions of the genome (Gasser 2002).

Observations of "free" ectopic regions of chromatin, exemplified by large $(\sim 15 \mathrm{~kb})$ circular plasmids in yeast underscore the context dependence of chromatin motion. An early study using a centromere-containing plasmid reported very limited movements, similar to those observed for centromeric regions in intact chromosomes (Marshall et al. 1997). However, more recently, Gartenberg et al. (2004) using plasmids designed to be either transcriptionally silent or competent, found that the active plasmids showed unconstrained movement, whereas the silent ones were strongly constrained and preferentially located near the nuclear envelope. Also relevant to the factors controlling intranuclear mobility is the finding that whereas double-stranded DNA breaks tend to stay together through the repair process (Kruhlak et al. 2006), the absence of a critical repair factor leads to long-range movements of the broken ends (Downs et al. 2004).

A general pattern emerging from numerous studies using different organisms is that all chromatin loci show constrained Brownian motion with rather similar diffusion constants, but a wide range of confinement volumes. A strong relationship between the confinement volume, and distance from the nuclear envelope has also been established, with loci closer to the envelope being more constrained. The periphery of the nucleus proximal to the nuclear envelope is considered a transcriptionally repressive environment, establishing a correlation between transcriptional silencing and limited intranuclear mobility (Marshall 2002). The underlying mechanism for this correlated behavior is not clear, but there is evidence that the constrained motion results from physical tethering to the nuclear lamina (Heun et al. 2001; Chubb et al. 2002; Gartenberg et al. 2004).

The higher spatial resolution $(\sim 20 \mathrm{~nm})$ of two-photon microscopy, together with a temporal resolution of $\sim 30 \mathrm{msec}$ has revealed new levels of complexity for constrained diffusive motion of chromatin (Levi et al. 2005). In these experiments, GFP-labeled repeats inserted in $\mathrm{CHO}$ cells showed periods of rapid constrained diffusive motion alternating with occasional energy-dependent curvilinear leaps of $\sim 150 \mathrm{~nm}$ that lasted only $0.3-2.0$ second. The energy-dependence of these leaps suggested that they may need the activity of chromatin remodeling complexes or other ATP-requiring changes in chromatin organization that could lead to events such as the decondensation of compact 30-nm fibers resulting in the rapid changes in location. Another important outcome of this study pertinent to the extent of physical coupling between neighboring loci on the same chromosome was the finding that loci 1-2 $\mu \mathrm{m}$ apart in the nucleus moved independently (Levi et al. 2005). A similar conclusion was reached by $\mathrm{Hu}$ et al. (2009) who showed that for a $\sim 2 \mathrm{Mb}$ region of chromatin that appeared as a linear cluster of beads in the light microscope, the individual beads moved independently. These findings suggest that neighboring loci are not structurally tied together by, for example, being anchored to an underlying nonchromatin structure. As it becomes possible to study yet closer regions of chromatin, there will be a point at which movement of the neighbors is correlated, and defining this point will be very useful in being able to infer the effective chromatin compaction level. Thus, although the accumulating data clearly demonstrate that chromatin in the interphase nucleus undergoes constant dynamic reorganization through constrained diffusive motion, the level(s) of chromatin higher-order structure that participate in this phenomenon remain to be determined. 
Some hints of the mechanism whereby loci undergo directed movement have emerged from work on an inserted transgene that typically resides in peripheral heterochromatin, but tends to relocate toward the center of the nucleus within 1-2 hour of transcriptional up-regulation (Chuang et al. 2006). Importantly, this movement was blocked by the expression of a mutant nuclear myosin I and by a nonpolymerizable mutant of actin. This intriguing finding should be viewed in the context that several families of actin and actinrelated proteins (ARPs) are found in the nucleus, many of which are components of chromatin remodeling and histone modification complexes (Chen and Shen 2007). Also, it has been shown that the BAF remodeling complex binds to the ends and branch points of actin filaments in a PIP2-dependent manner (Rando et al. 2002). Unlike their cytoplasmic counterparts, nuclear actins and ARPs tend to occur as monomers rather than the filamentous structures, and it is therefore curious that it was an actin polymerization mutant that was defective in long range movement (Chuang et al. 2006). Determining the mechanism by which actin/myosin influences the mobility and directionality of loci is clearly an important future goal.

\section{CHROMATIN DYNAMICS AND TRANSCRIPTION}

Studies of the spatial locations and transcriptional competence of loci with respect to their chromosome territories has provided some important insights. For example, the ANT2 gene located on the $\mathrm{X}$ chromosome is found in the interior of the territory of the inactive homolog, but peripherally on the active homo$\log$ (Dietzel et al. 1999). In contrast, the related ANT3 gene, which is located on the pseudoautosomal region of the $\mathrm{X}$ chromosome was peripherally located on the territory of both active and inactive homologs. This pattern of inactive genes located inside chromosome territories, and relocating to the periphery, perhaps by a "looping out" mechanism requiring a dramatic relaxation of local chromatin structure,
Chromatin Higher-order Structure and Dynamics

has been widely observed (Chambeyron and Bickmore 2005; Chambeyron et al. 2005; Volpi et al. 2000). Other studies have documented large scale movements of loci undergoing changes in transcriptional status as part of a developmental program. Loci were found to relocate to specific regions of the nucleus rather than locations within territories. An informative example is provided by the nuclear reorganization that accompanies differentiation of mouse lymphocytes (Brown et al. 1997, 1999). Here, lymphoid genes targeted for shutdown become relocated close to the heterochromatic clusters of pericentromeric chromatin characteristic of murine cells. Importantly, in this case, transcriptional repression appears to precede movement, suggesting that relocation is not a prerequisite for silencing. The relocation of genes destined for transcriptional up-regulation away from centromeric chromatin (Schübeler et al. 2000) or the nuclear periphery (Kosak et al. 2002) has also been documented.

Although there is a consensus regarding the correlation of intranuclear and intraterritory location with transcription, the chromatin conformational changes that accompany switches in transcriptional status are still far from clear. On the one hand, it seems unlikely that the bulky transcription machinery could access DNA and operate in compact chromatin, and in this respect, the concept of the chromatin looping out from compact domains to facilitate transcription is attractive. Indeed, transcriptional activity in LBC loops and polytene puffs is demonstrably unfolded, in some cases to the beads-on-a-string primary structure. Although there is general agreement that segments of chromatin are more compact when transcriptionally active than when silenced (e.g., Muller et al. 2001), the extent of this decondensation is unlikely to be as great as in LBC loops. A recent study designed to examine the compaction state of active and inactive chromatin in interphase nuclei has indicated quite small transcription-related changes in higher-order structure, implying that transcription can proceed in quite compact chromatin ( $\mathrm{Hu}$ et al. 2009). Labeled $\sim 2 \mathrm{Mb}$ chromatin domains designed to support a level of transcriptional 
activity typical of nuclear genes were inserted into cells, and tracked over time. In the uninduced state, the arrays assumed a linear, often beaded conformation, with lengths indicative of DNA packing ratios of over 1000:1. On transcriptional induction, the arrays retained their linear, beaded conformation, and showed only a modest 1.5- to threefold increase in length. No evidence was seen for large-scale looping or unfolding to the primary beads-on-a-string level of chromatin structure. Can transcription proceed in a compact structure that is over 10-times more compact than a $30-\mathrm{nm}$ fiber? In terms of access, several studies have shown that large macromolecules can penetrate quite compact chromatin (Verschure et al. 2003; Chen et al. 2005; Bancaud et al. 2009), perhaps as a function of the dynamic mobility of chromatin. Also, in a simple in vitro system, conformational fluctuations allowed direct access to the central nucleosome in a compact trinucleosome (Poirier et al. 2009). It is also possible that the binding of transcription factors could be followed by extensive, local unfolding, undetectable at the resolution of the light microscope.

The many facets of chromatin dynamics span orders of magnitude both in the spatial and temporal domains. Placing this complexity in the context of the level(s) of chromatin higher-order structure that are modulated during changes in transcriptional status remains an important challenge in understanding nuclear function. The difficulty is compounded by the current uncertainty regarding chromatin organization above the 30-nm fiber level. Nevertheless, continuing improvements in the spatial resolution with which specific loci can be imaged should soon begin to narrow the possible interpretations in terms of the level(s) of chromatin structure that could be involved. For example, as it becomes possible to image closer and closer neighboring loci on the same chromatin and follow their individual dynamics, there should be a point at which coupled motion will be observed. Once that point is reached, it will become possible to combine knowledge of the physical properties of arrays of nucleosomes with the observed dynamics and decide whether, for example, the data are consistent with a change from a compact to a decondensed 30-nm fiber. New strategies that allow "super" resolution in light microscopy will soon be in common use and may open the way to this type of correlative work.

\section{CONCLUDING REMARKS}

The past few years have witnessed remarkable progress in our understanding of chromatin higher-order structure, and this rapid trajectory can be expected to continue. There are some areas of research that seem especially poised to generate significant new insights in the near future, and others that have been overly neglected. Approaches that are likely to provide important new information in the near future include the application of "super resolution" light microscopy, and further exploration of the role of RNAi and other small and long nuclear RNAs (for a review see Wilusz et al. 2009).

A plethora of strategies for extending the resolution limitation of the light microscope well beyond the "diffraction limit" is maturing and some have been implemented commercially (Huang et al. 2009). Techniques capable of generating high resolution 3D images of the nuclear interior using principles such as PALM and STED will be especially suitable for studies of nuclei and chromatin. However, as experience with electron microscopy shows, high resolution alone may not be informative, especially for compact chromatin. Progress in understanding the paths of putative $30-\mathrm{nm}$ fibers in nuclei will require both high resolution and a contrasting technique that highlights a single stretch of DNA/chromatin. One application of an interference-based subdiffraction technique that was focused on the nuclear envelope showed the remarkable improvement that can be expected (Schermelleh et al. 2008), but little additional detail was seen in peripheral heterochromatin. It is also important that, as resolution improves further, preparative methods that provide a level of preservation consistent with the spatial resolution of the imaging system. Such methods should be capable of 
preserving membranous and cytoskeletal elements, as judged by electron microscopy.

Since the discovery of RNAi and other small nuclear RNAs, their importance in a host of nuclear functions has been shown. Among these functions is a role in the formation of the constitutive heterochromatin that flanks centromeres (Verdel et al. 2004; Moazed et al. 2006; Li et al. 2009). The process involves an RNAi-containing complex (RNA-induced transcriptional silencing, RITS) that binds to specific DNA sequences via siRNA-directed base pairing, and also to histone $\mathrm{H} 3$ methylated at lysine 9 , a heterochromatin-specific histone modification. In combination, these processes promote the spreading of heterochromatin. It seems very likely that, as the roles of small nuclear RNAs become more fully understood, they will be found to impact other aspects of chromatin higher-order structure. In addition, the identification and characterization of long noncoding RNAs that are retained in the nucleus is beginning to emerge as an important field that will add to our understanding of gene expression (see Wilusz et al. 2009 for a recent review).

In the category of neglected areas that merit further study are the ionic composition of nuclei, chromosomes, and chromatin, and the perhaps related issue of the impact of intranuclear molecular "crowding" (Hancock 2004a). Given the dramatic effects of changes in conformation that isolated chromatin undergoes in response to changes in the ionic environment, it is surprising that so little is known about the intranuclear ionic environment. It is generally assumed that "physiological" salt includes $\sim 100 \mathrm{mM}$ monovalent ions, and a smaller concentration $(\sim 1-2 \mathrm{mM})$ of divalent cations. Buffers for work with isolated nuclei and chromatin in solution typically include $\mathrm{Na}^{+}$or $\mathrm{K}^{+}$ for the monovalent cation, $\mathrm{Mg}^{++}$for the divalent cation, and $\mathrm{Cl}^{-}$for the anion. It is also well established that polyamines, especially spermidine $(4+)$ and spermine $(8+)$ are ubiquitous nuclear components with potent chromatin compaction properties. Very little is known about the intranuclear distributions and concentrations of these charged species, making it
Chromatin Higher-order Structure and Dynamics

difficult to have confidence that in vitro experiments and modeling studies are based on milieus that accurately reflect conditions in the nucleus. An isolated report of the distribution of cations in nuclei and isolated mitotic chromosomes using secondary ion mass spectrometry (Strick et al. 2001) concluded that during the transition from interphase to mitosis, $\mathrm{Ca}^{++}$and $\mathrm{Mg}^{++}$concentrations increased $\sim$ three- to four-fold, reaching $\sim 18 \mathrm{mM}$ and $\sim 11 \mathrm{mM}$ respectively, whereas isolated chromosomes contained almost twice these amounts. Interestingly, $\mathrm{Ca}^{++}$, but not $\mathrm{Mg}^{++}$ showed an axial enrichment in chromosomes. Of the monovalent ions, nuclear $\mathrm{Na}^{+}$concentrations were close to those measured in the cytosol, whereas $\mathrm{K}^{+}$was clearly enriched in both interphase and mitosis. It is not clear what the effect would be of using these remarkably high divalent ion levels in nuclear buffers, and it is unfortunate that the technique has not been further exploited using a wider range of ionic conditions.

An area of research that has received little attention until recently, but may have a farreaching impact on our understanding of chromatin function and dynamics relates to the highly crowded nuclear milieu (Hancock 2004a; Richter et al. 2008; Miyoshi and Sigimoto 2008; Bancaud et al. 2009). It is clear that the extremely high concentrations of macromolecules (of the order of $100 \mathrm{mg} / \mathrm{mL}$ ) that are present in the nucleus have important thermodynamic implications (Minton, 2001). These concentrations cannot be effectively recapitulated in vitro, with the consequence that experiments in solution may not reflect the events that occur in nucleo. In one set of experiments, when isolated nuclei were allowed to swell in low ionic strength medium, nucleoli and PML bodies disassembled and nucleolar transcription was dramatically reduced (Hancock 2004b). However, after adding inert macromolecules (polyethylene glycol or dextran) to $12 \% \mathrm{w} / \mathrm{v}$, the compartments reassembled, and nucleolar transcription recovered. Interestingly, this experiment also showed that it was not the lack of ions per se that caused disassembly. A fuller understanding of these effects, as well as the 


\section{C.L. Woodcock and R.P. Ghosh}

intranuclear levels and roles of ions is likely to expand our understanding of chromatin and nuclear organization.

This snapshot in time of chromatin higherorder structure and dynamics reveals an exciting period in research, with rapid advances being made on many fronts. As the different interweaving threads begin to coalesce, we can anticipate a much more comprehensive understanding of the structural and functional organization of chromatin in the context of its nuclear location. An overriding theme is that chromatin and its higher-order structures feature plasticity and dynamics rather than rigidity. How this is possible in what must at some level be a self-organizing system (Misteli 2001) remains a key open question.

\section{REFERENCES}

Arya G, Schlick T. 2006. Role of histone tails in chromatin folding revealed by a mesoscopic oligonucleosome model. Proc Natl Acad Sci 103: 16236-16241.

Bancaud A, Huet S, Daigle N, Mozziconacci J, Beaudouin J, Ellenberg J. 2009. Molecular crowding affects diffusion and binding of nuclear proteins in heterochromatin and reveals the fractal organization of chromatin. EMBO J doi:10.1038/emboj.2009.340.

Bednar J, Horowitz RA, Grigoryev SA, Carruthers LM, Hansen JC, Koster AJ, Woodcock CL. 1998. Nucleosomes, linker DNA, and linker histone form a unique structural motif that directs the higher-order folding and compaction of chromatin. Proc Natl Acad Sci 95: 14173-14178.

Belmont AS. 2006. Mitotic chromosome structure and condensation. Curr Opin Cell Biol 18: 632-638.

Belmont AS, Bruce K. 1994. Visualization of G1 chromosomes: A folded, twisted, supercoiled chromonema model of interphase chromatid structure. J Cell Biol 127: $287-302$.

Belmont AS, Braunfeld MB, Sedat JW, Agard DA. 1989. Large-scale chromatin structural domains within mitotic and interphase chromosomes in vivo and in vitro. Chromosoma 98: 129-43.

Belmont AS, Li G, Sudlow G, Robinett C. 1999. Visualization of large-scale chromatin structure and dynamics using the lac operator/lac repressor reporter system. Methods Cell Biol 58: 203-222.

Brown KE, Baxter J, Graf D, Merkenschlager M, Fisher AG. 1999. Dynamic repositioning of genes in the nucleus of lymphocytes preparing for cell division. Mol Cell 3: 207-17.

Brown KE, Guest SS, Smale ST, Hahm K, Merkenschlager M, Fisher AG. 1997. Association of transcriptionally silent genes with Ikaros complexes at centromeric heterochromatin. Cell 91: 845-54.
Buchberger JR, Onishi M, Li G, Seebacher J, Rudner AD, Gygi SP, Moazed D. 2008. Sir3-nucleosome interactions in spreading of silent chromatin in Saccharomyces cerevisiae. Mol Cell Biol 28: 6903-6918.

Chambeyron S, Bickmore WA. 2004. Chromatin decondensation and nuclear reorganization of the HoxB locus upon induction of transcription. Genes Dev 18: 1119-1130.

Chambeyron S, Da Silva NR, Lawson KA, Bickmore WA. 2005. Nuclear re-organisation of the Hoxb complex during mouse embryonic development. Development 132: 2215-2223.

Chen M, Shen X. 2007. Nuclear actin and actin-related proteins in protein dynamics. Curr Opin Cell Biol 19: 326-330.

Chen D, Dundr M, Wang C, Leung A, Lamond A, Misteli T, Huang S. 2005. Condensed mitotic chromatin is accessible to transcription factors and chromatin structural proteins. J Cell Biol 168: 41-54.

Cremer T, Cremer C, Baumann H, Luedtke EK, Sperling K, Teuber V, Zorn C. 1982. Rabl's model of the interphase chromosome arrangement tested in Chinese hamster cells by premature chromosome condensation and laser-UV-microbeam experiments. Hum Genet 60: $46-56$.

Chuang CH, Carpenter AE, Fuchsova B, Johnson T, de Lanerolle P, Belmont AS. 2006. Long-range directional movement of an interphase chromosome site. Curr Biol 16: $825-31$.

Chubb JR, Boyle S, Perry P, Bickmore WA. 2002. Chromatin motion is constrained by association with nuclear compartments in human cells. Curr Biol 12: 439-445.

Clapier CR, Cairns BR. 2009. The biology of chromatin remodeling complexes. Annu Rev Biochem 78: 273-304.

Clark DJ, Kimura T. 1990. Electrostatic mechanism of chromatin folding. J Mol Biol 211: 883-96.

Cook PR. 1999. The organization of transcription and replication. Science 284: 1790-1795.

Cook PR, Bazell IA. 1975. Supercoils in human DNA. J Cell Sci 19: 261-279.

Courbet S, Gay S, Arnoult N, Wronka G, Anglana M, Brison O, Debatisse M. 2008. Replication fork movement sets chromatin loop size and origin choice in mammalian cells. Nature 455: 557-560.

Cui Y, Bustamante C. 2000. Pulling a single chromatin fiber reveals the forces that maintain its higher-order structure. Proc Natl Acad Sci 97: 127-32.

Daban J-R. 2000. Physical constraints in the condensation of eukaryotic chromosomes. Local concentration of DNA versus linear packing ratio in higher order chromatin structures. Biochemistry 39: 3861-3866.

Daban J-R. 2003. High concentration of DNA in condensed chromatin. Biochem Cell Biol 81: 91-99

DiBoni U, Mintz AH. 1986. Curvilinear, 3-dimensional motion of chromatin domains and nucleoli in neuronal interphase nuclei. Science 234: 863-866.

Dietzel S, Schiebel K, Little G, Edelmann P, Rappold GA, Eils R, Cremer C, Cremer T. 1999. The 3D positioning of ANT2 and ANT3 genes within female X chromosome territories correlates with gene activity. Exp Cell Res 252: $363-375$. 
Dorigo B, Schlach T, Kulangara A, Duda S, Schroeder RR, Richmond TJ. 2004. Nucleosome arrays reveal the two-start organization of the chromatin fiber. Science 306: $1571-1573$

Downs JA, Jackson SP. 2004. A means to a DNA end: The many roles of Ku. Nature Rev Mol Cell Biol 5: 367-378.

Earnshaw WC, Laemmli UK. 1983. Architecture of metaphase chromosomes and chromosome scaffolds. J Cell Biol 96: 84-93.

Eltsov M, Maclellan KM, Maeshima K, Frangakis AS, Dubochet J. 2008. Analysis of cryo-electron microscopy images does not support the existence of 30 -nm chromatin fibers in mitotic chromosomes in situ. Proc Natl Acad Sci 105: 19732-19737.

Fan Y, Nikitina T, Morin-Kensicki EM, Zhao J, Magnuson TR, Woodcock CL, Skoultchi AI. 2003. H1 linker histones are essential for mouse development and affect nucleosome spacing in vivo. Mol Cell Biol 23: 4559-72.

Fan JY, Rangasamy D, Luger K, Tremethick DJ. 2004. H2A.Z alters the nucleosome surface to promote HP1alphamediated chromatin fiber folding. Mol Cell 16: 655-61.

Fischle W, Wang Y, Jacobs SA, Kim Y, Allis CD, Khourasanizadeh S. 2003. Molecular basis for the discrimination of repressive methyl-lysine marks in histone $\mathrm{H} 3$ by Polycomb and HP1 chromodomains. Genes Dev 17: $1870-1881$.

Gall JG. 1981. Chromosome structure and the C-value paradox. J Cell Biol 91: 3s-14s.

Gall J, Murphy C. 1998. Pictures in cell biology. Lampbrush chromosomes from sperm chromatin. Trends Cell Biol 8: 207.

Gartenberg MR, Neumann FR, Laroche T, Blaszczyk M, Gasser SM. 2004. Sir-mediated repression can occur independently of chromosomal and subnuclear contexts. Cell 119: 955-67.

Gaspar-Maia A, Alajem A, Polesso F, Sridharan R, Mason MJ, Heidersbach A, Ramalho-Santos J, McManus MT, Plath K, Meshorer E, et al. 2009. Chd1 regulates open chromatin and pluripotency of embryonic stem cells. Nature 460: 863-8.

Gasser SM, 2002, Visualizing chromatin dynamics in interphase nuclei. Science 296: 1412-1416.

Gasser SM, Laroche T, Falquet J, Boy de la Tour E, Laemmli UK. 1986. Metaphase chromosome structure. Involvement of topoisomerase II. J Mol Biol 188: 613-29.

Gondor A, Ohlsson R. 2009. Chromosome cross-talk in three dimensions. Nature 461: 199-205.

Greaves IK, Rangasamy D, Ridgway P, Tremethick DJ. 2007. H2A.Z contributes to the unique 3D structure of the centromere. Proc Natl Acad Sci 104: 525-530.

Grigoryev SA, Arya G, Correll S, Woodcock CL, Schlick T. 2009. Evidence for heteromorphic chromatin fibers from analysis of nucleosome interactions. Proc Natl Acad Sci 106: 13317-22.

Hagstrom KA, Meyer BJ. 2003. Condensin and cohesin: More than chromosome compactor and glue. Nat Rev Genet 4: 520-534.

Hake SB, Allis CD. 2006. Histone H3 variants and their potential role in indexing mammalian genomes: The "H3 barcode hypothesis." Proc Natl Acad Sci 103: $6428-35$.
Chromatin Higher-order Structure and Dynamics

Hancock R. 2004a. A role for molecular crowding effects in the assembly and function of compartments in the nucleus. J Struct Biol 146: 281-290.

Hancock R. 2004b. Internal organisation of the nucleus: Assembly of compartments by macromolecular crowding and the nuclear matrix model. Biol Cell 96: 595-601.

Hartl TA, Smith HF, Bosco G. 2008. Chromosome alignment and transvection are antagonized by condensin II. Science. 322: 1384-7.

Heitz E. 1928. Das Heterochromatin der Moose, 1, Jahrb Wiss Bot 69: 762-818.

Heun P, Laroche T, Shimada K, Furrer P, Gasser SM. 2001. Chromosome dynamics in the yeast interphase nucleus. Science 294: 2181-2186.

Hines KA, Cryderman DE, Flannery KM, Yang H, Vitalini MW, Hazelrigg T, Mizzen CA, Wallrath L. 2009. Domains of HP1 Required for Drosophila melanogaster heterochromatin spreading. Genetics. 182: 967-77.

Hirano T. 2006. At the heart of the chromosome: SMC proteins in action. Nat Rev Mol Cell Biol 7: 311-322.

Hirano T, Mitchison. 1993. Topoisomerase II does not play a scaffolding role in the organization of mitotic chromosomes assembled in Xenopus egg extracts. J Cell Biol 120: 601-612.

Horowitz RA, Agard DA, Sedat JM, Woodcock CL. 1994. The three dimensional archeitecture of chromatin in situ: Electron tomography reveals fibers composed of a continuously variable zigzag nucleosomal ribbon. J Cell Biol 125: 1-10.

Houchmandzadeh B, Marko JF, Chatenay D, Libchaber A. 1997. Elasticity and structure of eukaryote chromosomes studied by micromanipulation and micropipette aspiration. J Cell Biol 139: 1-12.

Hu Y, Kireev I, Plutz M, Ashourian N, Belmont AS. 2009. Large-scale chromatin structure of inducible genes: Transcription on a condensed, linear template. J Cell Biol 185: 87-100.

Huang B, Bates M, Zhuang X. 2009. Super-resolution fluorescence microscopy. Annu Rev Biochem 78: 993-1016.

Huebner MR, Spector DL. 2010. Chromatin dynamics. In Press.

Jacobs SA, Khorasanizadeh S. 2002. Structure of the HP1 chromodomain bound to a lysine 9-methylated histone H3 tail. Science 295: 2080-2083.

Jenuwein T, Allis CD. 2001. Translating the histone code. Science 293: 1074-80.

Jiang C, Pugh BF. 2009. Nucleosome positioning and gene regulation: Advances through genomics. Nat Rev Genet 10: $161-72$.

Kadauke S, Blobel GA. 2009. Chromatin loops in gene regulation. Biochim Biophys Acta 1789: 17-25.

Kireev I, Lakonishok M, Liu W, Joshi VN, Powell R, Belmont AS. 2008. In vivo immunogold labeling confirms largescale chromatin folding motifs. Nat Methods 5:311-313.

Kireeva N, Lakonishok M, Kireev I, Hirano T, Belmont AS. 2004. Visualization of early chromosome condensation: A hierarchical folding, axial glue model of chromosome structure. J Cell Biol 166: 775-785.

König P, Braunfeld MB, Sedat JW, Agard DA. 2007. The three-dimensional structure of in vitro reconstituted 


\section{C.L. Woodcock and R.P. Ghosh}

Xenopus laevis chromosomes by EM tomography. Chro mosoma 116: 349-372.

Kosak ST, Skok JA, Medina KL, Riblet R, Le Beau MM, Fisher AG, Singh H. 2002. Subnuclear compartmentalization of immunoglobulin loci during lymphocyte development. Science 296: 158-62.

Kouzarides T. 2007. Chromatin modifications and their function. Cell 128: 693-705.

Kruhlak MJ, Celeste A, Nussenzweig A. 2006. Spatiotemporal dynamics of chromatin containing DNA breaks. Cell Cycle 5: 1910-1912.

Kruithof M, Chien FT, Routh A, Logie C, Rhodes D, van Noort J. 2009. Single-molecule force spectroscopy reveals a highly compliant helical folding for the 30 -nm chromatin fiber. Nat Struct Mol Biol 16: 534-40.

Li H, Motamedi MR, Yip C K, Wang Z, Waltz T, Patel DJ, Moazed D. 2009. An Alpha Motif at Tas3 C Terminus Mediates RITS cis Spreading and Promotes Heterochromatic Gene Silencing. Mol Cell 34: 155-167.

Lieberman-Aiden E, van Berkum NL, Williams L, Imakaev M, Ragoczy T, Telling A, Amit I, Lajoie BR, Sabo PJ, Dorschner MO, et al. 2009. Comprehensive mapping of long-range interactions reveals folding principles of the human genome. Science 326: 289-93.

Levi V, Ruan Q, Plutz M, Belmont AS, Gratton E. 2005, Chromatin dynamics in interphase cells revealed by tracking in a two-photon excitation microscope. Biophys J 89: 4275-85.

Liaw H, Lustig AJ. 2006. Sir3 C-terminal domain involvement in the initiation and spreading of heterochromatin. Mol Cell Biol 26: 7616-7631.

Lohka MJ, Masui Y. 1983. Formation in vitro of sperm pronuclei and mitotic chromosomes induced by amphibian ooplasmic components. Science 220: 719-721.

Lu X, Wontakal SN, Emelyanov AV, Morcillo P, Konev AY, Fyodorov DV, Skoultchi AI. 2009. Linker histone H1 is essential for Drosophila development, the establishment of pericentric heterochromatin, and a normal polytene chromosome structure. Genes Dev 23: 452-65.

Luger K, Hansen JC. 2005. Nucleosome and chromatin fiber dynamics. Curr Opin Struct Biol 15: 188-196.

Luger K, Mader AW, Richmond RK, Sargent DF, Richmond TJ. 1997. Crystal structure of the nucleosome core particle at 2.8 A resolution. Nature 389: 251-60.

Mani RS, Tomlins SA, Callahan K, Ghosh A, Nyati MK, Varambally S, Palanisamy N, Chinnaiyan AM. 2009. Induced chromosomal proximity and gene fusions in prostate cancer. Science 326: 1230.

Marko JF. 2008. Micromechanical studies of mitotic chromosomes. Chromosome Res 16: 469-497.

Marko M, Hsieh C, Salmon N, Rodriguez M, Frank J, Manella C. 2008. FIB milling of frozen hydrated cells and tissues for TEM cryo-tomography. Microsc Microanal 13: 968CD.

Marko M, Hsieh C, Schalek R, Frank J, Mannella C. 2007. Focused-ion-beam thinning of frozen-hydrated biological specimens for cryo-electron microscopy. Nat Methods 4: $215-7$.

Maresca TJ, Heald R. 2006. The long and the short of it: Linker histone $\mathrm{H} 1$ is required for metaphase chromosome compaction. Cell Cycle 5: 589-91.
Marshall WF. 2002. Order and disorder in the cell nucleus. Curr Biol 12: R185-R192.

Marshall WF, Straight A, Marko JF, Swedlow J, Dernberg A, Belmont A, Murray AW, Agard DA, Sedat J. 1997. Interphase chromosomes undergo constrained diffusional motion in living cells. Curr Biol 7: 930-939.

Minton AP. 2001. The influence of macromolecular crowding and macromolecular confinement on biological reactions in physiological media. J Biol Chem 276: 10577-10580.

Misteli T. 2001. The concept of self-organization in cellular architecture. J Cell Biol 155: 181-185.

Miyoshi D, Sigimoto N. 2008. Molecular crowding effects on structure and stability of DNA. Biochimie 90 $1040-1051$.

Moazed D, Bühler M, Buker SM, Colmenares SU, Gerace EL, Gerber SA, Hong EJ, Motamedi MR, Verdel A, Villén J, Gygi SP. 2006. Studies on the mechanism of RNAidependent heterochromatin assembly. Cold Spring Harb Symp Quant Biol 71: 461-471.

Muller WG, Rieder D, Kreth G, Cremer C, Trajanoski Z, McNally JG. 2004. Generic features of tertiary chromatin structure as detected in natural chromosomes. Mol Cell Biol 24: 9359-9370.

Muller WG, Walker D, Hager G, McNally J. 2001. Large-scale chromatin decondensation and recondensation regulated by transcription from a natural promoter. J Cell Biol 154: $33-48$.

Nolis IK, McKay DJ, Mantouvalou E, Lomvaradas S, Merika M, Thanos D. 2009. Transcription factors mediate longrange enhancer-promoter interactions. Proc Natl Sci 106: $20222-7$.

Pederson T. 2000. Half a century of "the nuclear matrix." Mol Biol Cell 11: 799-805.

Phair RD, Misteli T. 2000. High mobility of proteins in the mammalian nucleus. Nature 404: 604-609.

Poirier MG, Marko JF. 2002. Mitotic chromosomes are chromatin networks without a mechanically contiguous protein scaffold. Proc Natl Acad Sci 99: 15393-15397.

Poirier MG, Marko JF. 2003. Micromechanical studies of mitotic chromosomes. Curr Top Dev Biol 55: 75-141.

Poirier MG, Oh E, Tims HS, Widom J. 2009. Dynamics and function of compact nucleosome arrays. Nat Struct Mol Biol 16: $938-44$.

Rando OJ, Zhao K, Janmey P, Crabtree GR. 2002. Phosphatidylinositol-dependent actin filament binding by the SWI/SNF-like BAF chromatin remodeling complex. Proc Natl Acad Sci 99: 2824-2829.

Richter K, Nessling M, Lichter P. 2008. Macromolecular crowding and its potential impact on nuclear function. Biochim Biophys Acta 1783: 2100-2107.

Robinson PJ, Fairall L, Huynh VA, Rhodes D. 2006. EM measurements define the dimensions of the " $30-\mathrm{nm}$ " chromatin fiber: Evidence for a compact, interdigitated structure. Proc Natl Acad Sci 103: 6506-6511.

Roix JJ, McQueen PG, Munson PJ, Parada LA, Misteli T. 2003. Spatial proximity of translocation-prone gene loci in human lymphomas. Nat Genet 34: 287-291.

Routh A, Sandin S, Rhodes D. 2008. Nucleosome repeat length and linker histone stoichiometry determine chromatin fiber structure. Proc Natl Acad Sci 105: 8872-8877. 
Rusk N. 2009. When ChIA PETs meet Hi-C. Nature Methods 6: 863 .

Santenard A, Torres-Padilla ME. 2009. Epigenetic reprogramming in mammalian reproduction: Contribution from histone variants. Epigenetics 4: 80-4.

Schermelleh L, Carlton PM, Haase S, Shao L, Winoto L, Kner P, Burke B, Cardoso MC, Agard DA, Gustafsson MG, et al. 2008. Subdiffraction multicolor imaging of the nuclear periphery with 3D structured illumination microscopy. Science 320: 1332-1336.

Schlach T, Duda S, Sargent DF, Richmond TJ. 2005. X-ray structure of a tetranucleosome and its implications for the chromatin fibre. Nature 436: 138-141.

Schübeler D, Francastel C, Cimbora DM, Reik A, Martin DI, Groudine M. 2000. Nuclear localization and histone acetylation: A pathway for chromatin opening and transcriptional activation of the human beta-globin locus. Genes Dev 14: $940-50$

Shelby RD, Hahn KM, Sullivan KF. 1996. Dynamic elastic behavior of alpha satellite domains visualized in living cells. J Cell Biol 135: 545-557.

Simpson RT. 1991. Nucleosome positioning: Occurrence, mechanisms, and functional consequences. Prog Nucleic Acid Res Mol Biol 40: 143-84.

Shogren-Knaak M, Ishii H, Sun JM, Pazin MJ, Davie JR, Peterson CL. 2006. Histone H4-K16 acetylation controls chromatin structure and protein interactions. Science 311: 844-847.

Shopland LS, Lynch CR, Peterson KA, Thornton K, Kepper N, Hase J, Stein S, Vincent S, Molloy KR, Kreth G, et al. 2006. Folding and organization of a contiguous chromosome region according to the gene distribution pattern in primary genomic sequence. J Cell Biol 174: 27-38.

Simpson RT, Thoma F, Brubaker J. 1985. Chromatin reconstituted from tandemly repeated cloned DNA fragments and core histones: A model system for study of higher order structure. Cell 42: 799-808.

Solovei I, Kreysing M, Lanctôt C, Kösem S, Peichl L, Cremer T, Guck J, Joffe B. 2009. Nuclear architecture of rod photoreceptor cells adapts to vision in mammalian evolution. Cell. 137: 356-68.

Soutoglou E, Misteli T. 2007. Mobility and immobility of chromatin in transcription and genome stability. Curr Opin Genet Dev 17: 435-4.

Strick R, Strissel PL, Gavrilov K, Levi-Setti R. 2001. Cation-chromatin binding as shown by ion microscopy is essential for the structural integrity of chromosomes. J Cell Biol 155: 899-910.

Strukov YG, Belmont AS. 2009. Mitotic chromosome structure: Reproducibility of folding and symmetry between sister chromatids. Biophys J 96: 1617-28.

Sullivan BA, Bickmore WA. 2000. Unusual chromosome architecture and behaviour at an HSR. Chromosoma 109: 181-9.
Chromatin Higher-order Structure and Dynamics

Suto RK, Clarkson MJ, Tremethick DJ, Luger K. 2000. Crystal structure of a nucleosome core particle containing the histone variant H2A.Z. Nature Struct Biol 7: 1121-1124.

Tremethick DJ. 2007. Higher-order structures of chromatin: The elusive $30 \mathrm{~nm}$ fiber. Cell 128: 651-654.

Trojer P, Reinberg D. 2007. Facultative heterochromatin: Is there a molecular signature. Mol Cell 28: 1-12.

Tumbar T, Sudlow G, Belmont AS. 1999. Large-scale chromatin unfolding and remodeling induced by VP16 acidic activation domain. J Cell Biol 145: 1341-54.

Vagnarelli P, Hudson DF, Ribeiro SA, Trinkle-Mulcahy L, Spence JM, Lai F, Farr CJ, Lamond AI, Earnshaw WC. 2006. Condensin and Repo-Man-PP1 co-operate in the regulation of chromosome architecture during mitosis. Nat Cell Biol 8: 1133-1142.

van Holde K. 1989. Chromatin. Springer Verlag, New York. van Holde K, Zlatanova J. 1995. Chromatin structure: Chasing a mirage? J Biol Chem 270: 8373-8376.

van Holde K, Zlatanova J. 2007. Chromatin fiber structure: Where is the problem now? Sem Cell Dev Biol 18: 651658.

Vazquez J, Bemont AS, Sedat JW. 2001. Multiple regimes of constrained chromosome motion are regulated in the interphase Drosophila nucleus. Curr Biol 11: 1227-1239.

Verdel A, Jia S, Gerber S, Sugiyama T, Gygi S, Grewal SI, Moazed D. 2004. RNAi-mediated targeting of heterochromatin by the RITS complex. Science 303: 672-676.

Verschure PJ, van der Kraan I, Manders EM, Hoogstraten D, Houtsmuller AB, van Driel R. 2003. Condensed chromatin domains in the mammalian nucleus are accessible to large macromolecules. EMBO Rep 4: 861-866.

Visel A, Rubin EM, Pennacchio LA. 2009. Genomic views of distant-acting enhancers. Nature 461: 199-205.

Volpi EV, Chevret E, Jones T, Vatcheva R, Williamson J, Beck S, Campbell RD, Goldsworthy M, Powis SH, Ragoussis J, et al. 2000. Large-scale chromatin organization of the major histocompatibility complex and other regions of human chromosome 6 and its response to interferon in interphase nuclei. J Cell Sci 113: 1565-2576.

Wilusz JE, Sunwoo H, Spector DL. 2009. Long non-coding RNAs: Functional surprises from the RNA world. Genes Dev 23: 1494-1504.

Woodcock CL. 2006. Chromatin architecture. Curr Opin Struct Biol 16: 213-220.

Woodcock CL, Dimitrov S. 2001. Higher-order structure of chromatin and chromosomes. Curr Opin Genet Dev 11: $130-135$.

Woodcock CL, Horowitz RA. 1995. Chromatin organization re-viewed. Trends Cell Biol 5: 272-277.

Woodcock CL, Skoultchi AI, Fan Y. 2006. Role of linker histone in chromatin structure and function: $\mathrm{H} 1$ stoichiometry and nucleosome repeat length. Chromosome Res 14: $17-25$. 


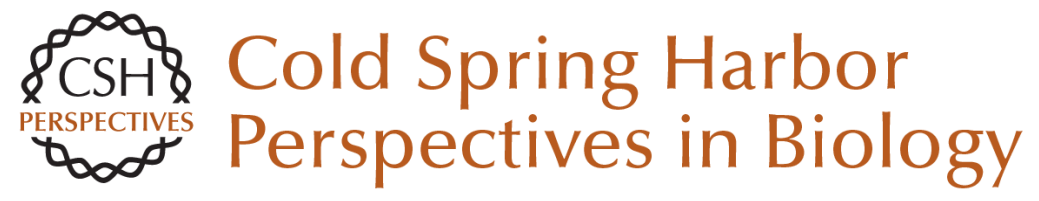

\section{Chromatin Higher-order Structure and Dynamics}

Christopher L. Woodcock and Rajarshi P. Ghosh

Cold Spring Harb Perspect Biol 2010; doi: 10.1101/cshperspect.a000596 originally published online April 7, 2010

\section{Subject Collection The Nucleus}

Nuclear Compartments: An Incomplete Primer to Nuclear Compartments, Bodies, and Genome Organization Relative to Nuclear Architecture Andrew S. Belmont

Uncovering the Principles of Genome Folding by 3D Chromatin Modeling

Asli Yildirim, Lorenzo Boninsegna, Yuxiang Zhan, et al.

3D or Not 3D: Shaping the Genome during Development Juliane Glaser and Stefan Mundlos

The Impact of Space and Time on the Functional Output of the Genome Marcelo Nollmann, Isma Bennabi, Markus Götz, et al.

Chromatin Mechanisms Driving Cancer

Berkley Gryder, Peter C. Scacheri, Thomas Ried, et al.

Liquid-Liquid Phase Separation in Chromatin Karsten Rippe

Mechanical Forces in Nuclear Organization Yekaterina A. Miroshnikova and Sara A. Wickström

Imaging Organization of RNA Processing within the Nucleus

Jeetayu Biswas, Weihan Li, Robert H. Singer, et al.
Mechanisms of Chromosome Folding and Nuclear Organization: Their Interplay and Open Questions Leonid Mirny and Job Dekker

Epigenetic Reprogramming in Early Animal Development

Zhenhai Du, Ke Zhang and Wei Xie

Essential Roles for RNA in Shaping Nuclear Organization

Sofia A. Quinodoz and Mitchell Guttman

The Molecular and Nuclear Dynamics of

X-Chromosome Inactivation

François Dossin and Edith Heard

Structure, Maintenance, and Regulation of

Nuclear Pore Complexes: The Gatekeepers of the

Eukaryotic Genome Marcela Raices and Maximiliano A. D'Angelo

The Nuclear Lamina Xianrong Wong, Ashley J. Melendez-Perez and Karen L. Reddy

The Nuclear Pore Complex as a Transcription Regulator Michael Chas Sumner and Jason Brickner

Physical Nature of Chromatin in the Nucleus Kazuhiro Maeshima, Shiori lida and Sachiko Tamura

For additional articles in this collection, see http://cshperspectives.cshlp.org/cgi/collection/

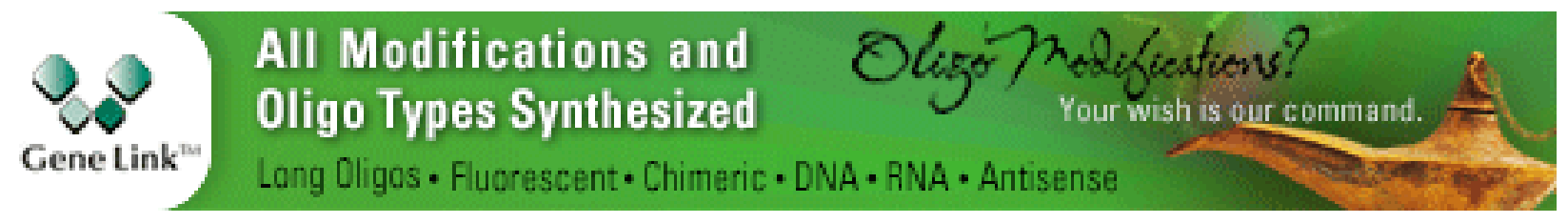


For additional articles in this collection, see http://cshperspectives.cshlp.org/cgi/collection/

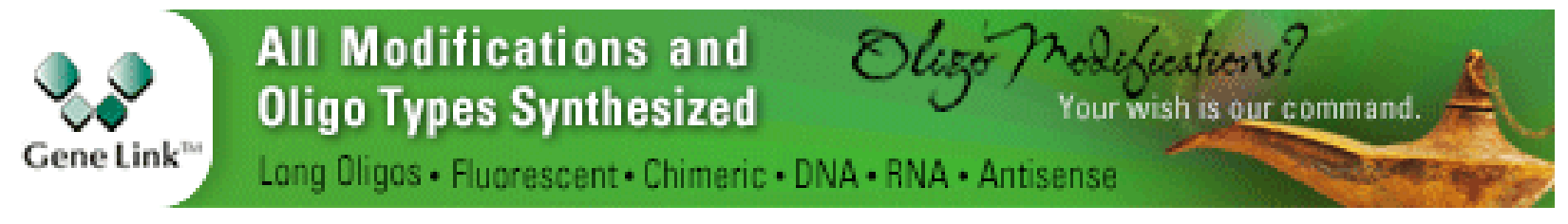

Copyright @ 2010 Cold Spring Harbor Laboratory Press; all rights reserved 